International Journal of Bifurcation and Chaos, Vol. 13, No. 4 (2003) 755-779

(c) World Scientific Publishing Company

\title{
CLUSTER SYNCHRONIZATION IN THREE-DIMENSIONAL LATTICES OF DIFFUSIVELY COUPLED OSCILLATORS
}

\author{
VLADIMIR N. BELYKH \\ Mathematics Department, Volga State Academy, 5, Nesterov St., \\ Nizhny Novgorod, 603600 Russia \\ IGOR V. BELYKH and MARTIN HASLER \\ Laboratory of Nonlinear Systems, \\ School of Computer and Communication Sciences, \\ Swiss Federal Institute of Technology (EPFL), \\ CH-1015 Lausanne, Switzerland \\ KONSTANTIN V. NEVIDIN \\ Volga State Academy, 5, Nesterov St., \\ Nizhny Novgorod, 603600 Russia
}

Received November 6, 2001; Revised December 20, 2001

\begin{abstract}
Cluster synchronization modes of continuous time oscillators that are diffusively coupled in a three-dimensional (3-D) lattice are studied in the paper via the corresponding linear invariant manifolds. Depending in an essential way on the number of oscillators composing the lattice in three volume directions, the set of possible regimes of spatiotemporal synchronization is examined. Sufficient conditions of the stability of cluster synchronization are obtained analytically for a wide class of coupled dynamical systems with complicated individual behavior. Dependence of the necessary coupling strengths for the onset of global synchronization on the number of oscillators in each lattice direction is discussed and an approximative formula is proposed. The appearance and order of stabilization of the cluster synchronization modes with increasing coupling between the oscillators are revealed for 2-D and 3-D lattices of coupled Lur'e systems and of coupled Rössler oscillators.
\end{abstract}

Keywords: Cluster synchronization; chaos; stability; 3-D lattice.

\section{Introduction}

Over the past decade, there has been significant interest in studying the behavior of coupled chaotic systems. Numerous studies of the spatiotemporal dynamics of ensembles of coupled oscillators have found different types of coherent structures, patterns and synchronization phenomena. Since early works concerned with a small number of coupled oscillators, the increasing interest in synchronization phenomena in dynamical systems has led many researchers to consider the synchronization phenomena in large lattices or networks of coupled chaotic oscillators.

The simplest mode of spatiotemporal behavior that can arise in a lattice of coupled identical oscillators with chaotic behavior is full synchronization. Here all oscillators of the lattice acquire identical chaotic behavior even though their initial conditions may be different. Since the pioneering works [Fujisaka \& Yamada, 1983; Afraimovich 
et al., 1986; Pecora \& Carroll, 1990], the study of full chaos synchronization remains of strong interest, see e.g. [Belykh et al., 1993; Heagy et al., 1994; Fradkov \& Pogromsky, 1998; Pecora \& Carroll, 1998; Fink et al., 2000; Josić, 2000] and the references therein. The phenomenon of chaos synchronization has many different potential applications. In engineering, for example, it is being considered as a tool for transmitting information by means of chaotic signals [Pecora \& Carroll, 1990; Bel'skii \& Dmitriev, 1993; Hasler, 1995; Chen, 2000].

Cluster synchronization is observed in a lattice when the oscillators synchronize in groups but there is no synchronization among the groups [Kaneko, 1990; Belykh \& Mosekilde, 1996; Xie \& Hu, 1997; Hasler et al., 1998; Belykh et al., 2000; Belykh et al., 2001]. Clustering is often defined in a broader sense as the emergence of large-scaled ordered coherent structures presenting different degrees of correlation between the interacting systems and accompanied by the temporal evolution of spatially averaged quantities [Lemaître \& Chaté, 1999; Rabinovich et al., 1999].

The synchronization phenomena in 2-D lattices of coupled oscillators have been usually studied through numerical analysis. Most analytical studies were concerned with 2-D lattices composed from one-dimensional bistable (or multistable) oscillators. The main interest in these studies was concentrated in equilibrium solutions and, particularly, in the spatial features presented by stable equilibria which are often called mosaic solutions [Chow \& Mallet-Paret, 1995; Mallet-Paret \& Chow, 1995, Thiran et al., 1995; Nekorkin et al., 1997; Thiran, 1997]. More recently, 2-D lattices of coupled chaotic continuous (or discrete-time) oscillators have received a great deal of attention [Afraimovich et al., 1997]. In particular, interesting spatio- temporal periodic patterns in a 2-D lattice of coupled logistic maps were found [Xie \& Hu, 1997], sufficient conditions for asymptotic synchronization in a 2-D lattice of coupled Lorenz systems were presented [Chiu et al., 2000]. Synchronization in 3-D lattices of locally coupled limit-cycle and chaotic oscillators has been also studied. Sherman [1994] considered, in particular, a $5 \times 5 \times 5$ lattice of locally coupled biological cell models and found numerically different types of inphase and antiphase synchronization between the cells, presenting different levels of correlation between the cells. Kazantsev et al. [2001] considered a three-dimensional structure composed of two coupled 2-D lattices of diffusively coupled limit-cycle oscillators and proved, in particular, the possibility of interlayer synchronization.

We have recently discovered a family of embedded linear invariant manifolds of a 1-D array of diffusively coupled identical dynamical systems, whether chaotic or periodic [Belykh et al., 2000]. Depending in an essential manner on the number of oscillators contained in the array and on boundary conditions, these invariant manifolds define the strict set of all possible modes of cluster synchronization that can occur in the 1-D lattice. The purpose of the present paper is to extend our results on the existence and stability of different cluster synchronization regimes for a 1-D chain of coupled systems to the cases of planar and volume lattices of diffusively coupled oscillators. We define cluster synchronization as a spatiotemporal regime where the oscillators involved into the same cluster have identical temporal dynamics and completely synchronize between each other. In this paper we do not consider the emergence of cluster patterns with "similar" averaged behavior.

We study in this paper a 3-D lattice of diffusively coupled identical oscillators that is described by the following dynamical system:

$$
\left\{\begin{aligned}
\dot{x}_{i, j, k}= & f\left(x_{i, j, k}, y_{i, j, k}\right)+\varepsilon_{1}\left(x_{i+1, j, k}-2 x_{i, j, k}+x_{i-1, j, k}\right)+\varepsilon_{2}\left(x_{i, j+1, k}-2 x_{i, j, k}+x_{i, j-1, k}\right) \\
& +\varepsilon_{3}\left(x_{i, j, k+1}-2 x_{i, j, k}+x_{i, j, k-1}\right) \\
\dot{y}_{i, j, k}= & g\left(x_{i, j, k}, y_{i, j, k}\right) \quad i=\overline{1, N}_{1}, j=\overline{1, N}_{2}, k=\overline{1, N}_{3}
\end{aligned}\right.
$$

with zero-flux $x_{i, j, 0} \equiv x_{i, j, 1}, x_{i, 0, k} \equiv x_{i, 1, k}, x_{0, j, k} \equiv$ $x_{1, j, k}, x_{i, j, N_{3}+1} \equiv x_{i, j, N_{3}}, x_{i, N_{2}+1, k} \equiv x_{i, N_{2}, k}$, and $x_{N_{1}+1, j, k} \equiv x_{N_{1}, j, k}$ or periodic boundary conditions (BC).

In the system (1), $x_{i, j, k} \in R^{1}$ is a scalar variable of the $m$-dimensional vector of the $(i, j, k)$-th oscillator variables, and $y_{i, j, k} \in R^{m-1}$ is an $m-1$ vector. $f\left(x_{i, j, k}, y_{i, j, k}\right): R^{m} \rightarrow R^{1}$ and $g\left(x_{i, j, k}, y_{i, j, k}\right): R^{m} \rightarrow R^{m-1}$ are scalar and vector functions, respectively. $m$ is the dimension of the individual oscillator, and $D=N_{1} \cdot N_{2} \cdot N_{3} \cdot m$ is the dimension of the whole lattice system (1). $\varepsilon_{1}>0, \varepsilon_{2}>0$ and $\varepsilon_{3}>0$ are coupling parameters 
determining the coupling strengths between the oscillators in three directions of the volume lattice. $N_{1}, N_{2}$ and $N_{3}$ define the size of the lattice.

Denote the $m$-vectors

$$
X_{i, j, k}=\left(\begin{array}{c}
x_{i, j, k} \\
y_{i, j, k}
\end{array}\right), \quad F=\left(\begin{array}{c}
f \\
g
\end{array}\right) .
$$

Omitting for simplicity the index $(i, j, k)$, we indicate only shifted ones such that $X_{i-1, j, k} \equiv X_{i-1}$, $X_{i+1, j, k} \equiv X_{i+1}, X_{i, j-1, k} \equiv X_{j-1}$, and so forth. Thus we can rewrite the system (1) in the alternative form

$$
\dot{X}=F(X)+\varepsilon_{1} P \triangle_{i}+\varepsilon_{2} P \triangle_{j}+\varepsilon_{3} P \triangle_{k},
$$

with zero-flux or periodic BC. In the system (2), the diffusive coupling terms are denoted by $\triangle_{l}=$ $\left(X_{l-1}-2 X+X_{l+1}\right), X=X_{l, j, k} ; X_{i, l, k} ; X_{i, j, l}$, where $l=i, j, k$, respectively, and

$$
P=\left(\begin{array}{cccc}
1 & 0 & \cdots & 0 \\
0 & 0 & \cdots & 0 \\
\vdots & \cdots & \ddots & \vdots \\
0 & \cdots & \cdots & 0
\end{array}\right)
$$

is the $m \times m$ matrix whose elements determine by which variables the oscillators are coupled.

Note that the system (1) represents a class of 3-D reaction-diffusion Cellular Nonlinear Networks (CNN) that may be considered as a universal active substrate or medium for modeling and generating many pattern formation and synchronization phenomena from numerous disciplines including biology, chemistry, ecology, engineering, physics, etc. [Chua \& Yang, 1988; Chua et al., 1995; Chua, 1997].

We have organized this paper as follows. In Sec. 2 the set of possible modes of cluster synchronization for both cases of the 2-D and 3-D lattices (1) is examined. Then, in Sec. 3, stability conditions of the synchronization manifolds for a wide class of dynamical systems are derived. Using these conditions, a conjecture on the estimation of threshold coupling constants required for the onset of global synchronization in 3-D lattices is made. Section 4 combines the theoretical study with numerical simulation of stable cluster synchronization in 2-D and 3-D lattices of coupled Lur'e systems. Finally, in Sec. 5, results of numerical simulations that show the stable cluster synchronization regimes with chaotic temporal dynamics in 2-D and 3-D lattices of coupled Rössler systems are presented.

\section{Existence of Cluster Synchronization Manifolds}

\subsection{Three-dimensional lattice}

Cluster synchronization of coupled oscillators is intimately related to linear invariant manifolds of the system (1).

Definition. Let the set of vertices of the 3-D lattice be decomposed into the disjoint subsets $V=$ $V_{1} \cup \cdots \cup V_{d}, V_{\gamma} \cap V_{\mu}=\emptyset$ given by the equalities of groups of the coordinates of oscillators. If the decomposition of the vectors is compatible with the system (1) then the linear subspace of $R^{D}$

$$
\begin{aligned}
M(d) \equiv & M\left(V_{1}, \ldots, V_{d}\right) \\
=\{ & \underline{X} \mid X_{i, j, k}=X_{i^{\prime}, j^{\prime}, k^{\prime}}, \\
& \text { if there exists a } \gamma \text { such that } \\
& \left.(i, j, k) \in V_{\gamma} \text { and }\left(i^{\prime}, j^{\prime}, k^{\prime}\right) \in V_{\gamma}\right\}
\end{aligned}
$$

is invariant under the dynamics given by Eq. (1) and is called a linear invariant manifold of the system (1). The coordinates in the manifold $M(d)$ are $U_{l}=X_{i_{l}, j_{l}, k_{l}}, l=1,2, \ldots, d . d$ is the number of clusters.

Let us introduce an $N_{1} \times N_{2} \times N_{3}$ volume matrix $A=\left[a_{i, j, k}\right]$, where the symbol $a_{i, j, k}$ denotes the oscillator standing in the $i$ th line, $j$ th column, and $k$ th vertical. We shall use the same symbol for oscillators of the same cluster. The definition implies that the $d$ base elements of the matrix $A$ disagree with each other and all the other $r=N_{1} \times N_{2} \times$ $N_{3}-d$ elements coincide with these $d$ base elements. At the same time, $r \times m$ equations in Eq. (1) become linearly dependent of the $d \times m$ base ones.

The numbers $q_{1}, q_{2}, \ldots, q_{d}$ of oscillators in each cluster satisfy the natural condition $\sum_{p=1}^{d} q_{p}=$ $N_{1} \times N_{2} \times N_{3}$.

We shall use the following notations.

(1) Three sets of planes in each of three lattice directions are $P^{l}=I^{i}, J^{j}, K^{k}$, where $l=i, j$ or $k, i=\overline{1, N}_{1}, j=\overline{1, N}_{2}, k=\overline{1, N}_{3}$, respectively. Define them as $I^{i}=\left\{(i, j, k) \mid j=\overline{1, N}_{2}\right.$, $\left.k=\overline{1, N}_{3}\right\}$.

(2) Each plane $P^{l}$ may be presented as a set of rows $R^{l}$ and columns $C^{l}$, so that $K^{k}=\bigcup_{i=1}^{N_{1}}\left\{R_{i}^{k}\right\}=$ $\bigcup_{j=1}^{N_{2}}\left\{C_{j}^{k}\right\}, J^{j}=\bigcup_{k=1}^{N_{3}}\left\{R_{k}^{j}\right\}=\bigcup_{i=1}^{N_{1}}\left\{C_{i}^{j}\right\}$, and $I^{i}=\bigcup_{j=1}^{N_{2}}\left\{R_{j}^{i}\right\}=\bigcup_{k=1}^{N_{3}}\left\{C_{k}^{i}\right\}$, where $C_{k}^{i}=$ $\left\{(i, j, k) \mid j=\overline{1, N}_{2}\right\}$. 
As for the single oscillators, we shall use the notations $P^{l_{1}}=P^{l_{2}}\left(R_{l_{1}}^{l}=R_{l_{2}}^{l}, C_{l_{1}}^{l}=C_{l_{2}}^{l}\right)$ for two synchronized planes (rows, columns).

Cluster synchronization in 3-D lattices generated by cluster synchronization of $1-D$ arrays can be presented by a code notation of the manifold

$$
\begin{aligned}
M(d)= & M\left(n_{1}, n_{2}, n_{3}\right) \\
= & {[\underbrace{r_{i_{1}}, \ldots, r_{i_{l}}, \ldots}_{N_{1}}\} \times\{\underbrace{c_{j_{1}}, \ldots, c_{j_{l}}, \ldots}_{N_{2}}\} } \\
& \left.\times\{\underbrace{v_{k_{1}}, \ldots, v_{k_{l}}, \ldots}_{N_{3}}\}\right],
\end{aligned}
$$

where each symbol $r_{i_{l}}, l=\overline{1, n}_{1} ; c_{j_{l}}, l=\overline{1, n}_{2}$; and $v_{k_{l}}, l=\overline{1, n}_{3}$ is repeated $q_{1}, \ldots, q_{n_{1}}\left(q_{n_{2}}\right)\left(q_{n_{3}}\right)$ times, respectively. That is, the place of each symbol $r_{i_{l}}, c_{j_{l}}$ and $v_{k_{l}}\left(l=1, n_{1} ; l=1, n_{2} ; l=1, n_{3}\right)$ in the subsets defines the place of each oscillator in the $i$ th, $j$ th and $k$ th lattice directions, respectively. Oscillators involved in one cluster along one direction of the volume lattice are denoted by a symbol with the same index. Thus, the oscillators in the same cluster are identified by the identical triple $\left(r_{i}, c_{j}, v_{k}\right)$.

As an example we introduce two well-known clusters.

(1) For $d=1$, the lattice (1) has the full synchronization manifold $M(1) \equiv M(1,1,1)=$ $\left\{X_{i, j, k} \equiv U_{1}\right\}$. Its code notation reads $M(1)=\left[\{\underbrace{r_{1}, r_{1}, \ldots, r_{1}}_{N_{1}}\} \times\{\underbrace{c_{1}, c_{1}, \ldots, c_{1}}_{N_{2}}\} \times\right.$ $\left.\{\underbrace{v_{1}, v_{1}, \ldots, v_{1}}_{N_{3}}\}\right]$. Dynamics in the hyperplane $M(1)$ is defined by the single oscillator and therefore we have a single homogeneous cluster exhibiting full synchronization in the volume lattice when the spatial sites are all doing the same thing at the same moment, even though it is chaotic motion.

(2) For $d=N_{1} \times N_{2} \times N_{3}$ we have the invariant manifold $M_{0}$, being the phase space of the system (1), $M_{0}=\left[\left\{r_{1}, r_{2}, \ldots, r_{N_{1}}\right\} \times\right.$ $\left.\left\{c_{1}, c_{2}, \ldots, c_{N_{2}}\right\} \times\left\{v_{1}, v_{2}, \ldots, v_{N_{3}}\right\}\right]$. Obviously, the lattice (1) has $d=N_{1} \times N_{2} \times N_{3}$ clusters defined by $N_{1} \times N_{2} \times N_{3}$ independent oscillators.

The coupling in the system (1) is free of crossing terms and therefore the group spatiotemporal behavior of the 2-D lattices $P^{l}$ is similar to that of the 1-D arrays in one lattice direction and it appears that the hierarchy and embeddings of cluster synchronization manifolds discovered for the 1-D array [Belykh et al., 2000] plays the crucial role in the present study of 2-D and 3-D lattices of coupled oscillators.

The volume lattice $A$ may be considered as a 1-D array of planes $P^{l}$ in each of three lattice directions, where the elements $\left(P^{1}, P^{2}, \ldots, P^{N}\right)=A$ $\left(N=N_{1} ; N_{2} ; N_{3}\right.$, respectively) introduce a set of 2-D lattices. Applying directly the results from [Belykh et al., 2000] for the set of 2-D lattices, we obtain the following assertion.

Proposition 2.1. Let the system (2) have either zero-flux or periodic BC. Then the following holds.

(1) There exists a symmetrical 2-D lattice invariant manifold $M_{s}^{r}\left(n_{1}, N_{2}, N_{3}\right)$ with $n_{1}=$ $\operatorname{int}\left(\left(N_{1}+1\right) / 2\right)$ defined by the equalities $\left\{X_{N_{1}-i+1, j, k}=X_{i, j, k}=U_{i, j, k}, i=1\right.$, $2, \ldots, \operatorname{int}\left(\left(N_{1}+1\right) / 2\right), j=1,2, \ldots, N_{2}$, $\left.k=1,2, \ldots, N_{3}\right\}$. The code notation of the manifold has the form $M_{s}^{r}\left(n_{1}, N_{2}, N_{3}\right)=$ $\left[\left\{r_{1}, r_{2}, \ldots, r_{n_{1}-1}, r_{n}, r_{n_{1}-1}, \ldots, r_{2}, r_{1}\right\} \times\right.$ $\left.\left\{c_{1}, c_{2}, \ldots, c_{N_{2}}\right\} \times\left\{v_{1}, v_{2}, \ldots, v_{N_{3}}\right\}\right]$ for odd number of oscillators $N_{1}=2 n_{1}-1$ composing the lattice in the ith direction, and the form $M_{s}^{r}\left(n_{1}, N_{2}, N_{3}\right)=\left[\left\{r_{1}, r_{2}, \ldots\right.\right.$, $\left.r_{n_{1}}, r_{n_{1}}, \ldots, r_{2}, r_{1}\right\} \times\left\{c_{1}, c_{2}, \ldots, c_{N_{2}}\right\} \times$ $\left.\left\{v_{1}, v_{2}, \ldots, v_{N_{3}}\right\}\right]$ for even $N_{1}=2 n_{1}$.

(2) The system (2) has an asymmetrical invariant manifold $M_{a}^{r}\left(n_{1}, N_{2}, N_{3}\right)$, if $N_{1}=$ $p_{1} \cdot n_{1}$ with $p_{1}$ and $n_{1}$ being arbitrary integers, defined by the equalities $\left\{X_{i, j, k}=\right.$ $X_{i+2 n_{1} l, j, k}, l=1,2, \ldots, \operatorname{int}\left(\left(p_{1}-1\right) / 2\right)$ and $X_{i, j, k}=X_{-i+1+2 n_{1} l, j, k}, l=1,2, \ldots, \operatorname{int}\left(p_{1} / 2\right)$, $i=1,2, \ldots, n_{1}, j=1,2, \ldots, N_{2}, k=$ $\left.1,2, \ldots, N_{3}\right\}$. The code notation of the manifold has the form $M_{a}^{r}\left(n_{1}, N_{2}, N_{3}\right)=$ $\left[\{\underbrace{r_{1}, r_{2}, \ldots, r_{n_{1}}, r_{n_{1}}, \ldots, r_{1}, r_{1}, \ldots, r_{n_{1}}, r_{n_{1}}, \ldots}_{p_{1} \cdot n_{1}}\} \times\right.$ $\left.\left\{c_{1}, c_{2}, \ldots, c_{N_{2}}\right\} \times\left\{v_{1}, v_{2}, \ldots, v_{N_{3}}\right\}\right]$.

(3) The system (2) has similar invariant manifolds in the $j$ th and $k$ th lattice directions: symmetrical manifolds $M_{s}^{c}\left(N_{1}, n_{2}, N_{3}\right)$, where $n_{2}=$ $\operatorname{int}\left(\left(N_{2}+1\right) / 2\right), M_{s}^{v}\left(N_{1}, N_{2}, n_{3}\right)$, where $n_{3}=$ $\operatorname{int}\left(\left(N_{3}+1\right) / 2\right)$, and asymmetrical synchronization manifolds $M_{a}^{c}\left(N_{1}, n_{2}, N_{3}\right)$ with $N_{2}=p_{2}$. $n_{2}$ and $M_{a}^{v}\left(N_{1}, N_{2}, n_{3}\right)$, where $N_{3}=p_{3} \cdot n_{3}$.

The separate oscillators of the 1-D array, involved in the cluster synchronization regime, are 
introduced here by the 2-D lattices in three lattice directions. That is, for example, in the case of odd $N_{1}=2 n_{1}-1$, the dynamics in $M_{s}^{r}\left(n_{1}, N_{2}, N_{3}\right)$ defines a cluster synchronization mode under which the 2-D lattices are synchronized in pairs around the middle lattice $I^{n_{1}}$ which has no pair and remains unsynchronized, i.e. $I^{1}=I^{N_{1}}, I^{2}=I^{N_{1}-1}$ and so forth.

Corollary. In the case of periodic $B C$ each element of the 3-D lattice may be considered as the first one in each lattice direction, and the system (1) has $N_{1}-1$ additional invariant manifolds $M_{r}^{s}$ and $M_{r}^{a}, N_{2}-1$ manifolds $M_{c}^{s}$ and $M_{c}^{a}$, and $N_{3}-1$ additional synchronization manifolds $M_{v}^{s}$ and $M_{v}^{a}$.

Proposition 2.2. Let oscillators of the volume lattice be synchronized in one, say in the kth, direction so that the 2-D lattices $K^{1}=K^{2}=\cdots=$ $K^{N_{3}} \equiv K$. Consider the 2-D lattice $K$ as a 1-D array of rows (columns) : $K=\left(R_{1}, R_{2}, \ldots, R_{N_{1}}\right)$ $\left(K=\left(C_{1}, C_{2}, \ldots, C_{N_{2}}\right)\right)$. Then cluster synchronization modes of the 1-D array of synchronized rows (columns) have in turn hierarchy and embeddings identical to the oscillators of the 1-D array.

Proposition 2.3. Let the volume lattice be synchronized in two, say in the $k$ th and $j$ th, directions then the lattice system is identical to 1-D lines in the ith direction.

One may interpret these two trivial assertions both as full synchronization of the $K$ planes and sequential synchronization of the columns $C_{j}$ of $K$, and synchronization of the $J$ planes with sequential synchronization of the rows $R_{k}$.

Proposition 2.4. The system (1) has a family of intersection synchronization manifolds $M_{s, a}^{\times}\left(n_{1}\right.$, $\left.n_{2}, n_{2}\right)=M_{s, a}^{r}\left(n_{1}, N_{2}, N_{3}\right) \cap M_{s, a}^{c}\left(N_{1}, n_{2}, N_{3}\right) \cap$ $M_{s, a}^{c}\left(N_{1}, N_{2}, n_{3}\right)$, being an intersection of any row, any column and any vertical synchronization manifolds existing in the case of the 1-D array. Thus, the manifold $M^{\times}\left(n_{1}, n_{2}, n_{3}\right)$ has the code notation $M^{\times}\left(n_{1}, n_{2}, n_{3}\right)=M^{r} \cap M^{c} \cap M^{v}$. The dimension of the manifold is $\operatorname{dim} M^{\times}=n_{1} \cdot n_{2} \cdot n_{3} \cdot m$, and $U_{i, j, k}$, with $i=\overline{1, n}_{1}, j=\overline{1, n}_{2}$ and $k=\overline{1, n}_{3}$ are coordinates in $M^{\times}\left(n_{1}, n_{2}, n_{3}\right)$.

The existence of the intersection synchronization manifolds $M^{\times}\left(n_{1}, n_{2}, n_{3}\right)$ implies that many cluster manifolds of the volume lattice (1) have the structure of a topological product of cluster synchronization manifolds existing in three lattice directions. For different composed numbers $N_{1}, N_{2}$, and $N_{3}$ the manifolds for $I^{i}, J^{j}, K^{k}$ may be rather complicated, hence the complexity of the volume clusters increases as a product.

While the product clusters are always present, there may be additional manifolds, depending on additional symmetries of the system. Let us consider a particular case of the system (1) with $N_{1}=$ $N_{2}=N_{3}=N$ and $\varepsilon_{1}=\varepsilon_{2}=\varepsilon_{3}=\varepsilon$. Thus we consider the cubic lattice (1) with equal coupling strengths in three lattice directions and zero-flux or periodic BC. Under these conditions, the following holds.

\section{Proposition 2.5}

(1) The system (1) has additional simple symmetries and is invariant under the involutions $\{i \leftrightarrow j\},\{i \leftrightarrow k\},\{j \leftrightarrow k\},\{(i, j) \leftrightarrow(N-j+$ $1, N-i+1)\},\{(j, k) \leftrightarrow(N-k+1, N-j+1)\}$, and $\{(i, k) \leftrightarrow(N-k+1, N-i+1)\}$. Therefore it has additional invariant manifolds $M_{i j}^{+}(d)=$ $\left\{X_{j, i, k}=X_{i, j, k}=U_{i, j, k}, i=\overline{1, N}, i \leq j \leq N\right.$, $k=\overline{1, N}\}, M_{j k}^{+}(d)=\left\{X_{i, k, j}=X_{i, j, k}=U_{i, j, k}\right.$, $i=\overline{1, N}, j=\overline{1, N}, j \leq k \leq N\}, M_{i k}^{+}(d)=$ $\left\{X_{k, j, i}=X_{i, j, k}=U_{i, j, k}, k \leq i \leq N, j=\overline{1, N}\right.$, $k=\overline{1, N}\}$, defining the symmetries with respect to the principal diagonal sections, respectively, and $M_{i j}^{-}(d)=\left\{X_{N-j+1, N-i+1, k}=X_{i, j, k}=\right.$ $\left.U_{i, j, k}, i=\overline{1, N}, 1 \leq j \leq N-i+1, k=\overline{1, N}\right\}$, $M_{j k}^{-}(d)=\left\{X_{i, N-k+1, N-j+1}=X_{i, j, k}=U_{i, j, k}\right.$, $i=\overline{1, N}, j=\overline{1, N}, 1 \leq k \leq N-j+1\}$, $M_{i k}^{-}(d)=\left\{X_{N-k+1, j, N-i+1}=X_{i, j, k}=U_{i, j, k}\right.$, $i=\overline{1, N}, j=\overline{1, N}, 1 \leq k \leq N-i+1$, determining synchronization of the oscillators with respect to the secondary diagonal sections, $d=$ $N^{2}(N+1) / 2$. Obviously, all possible intersections of these manifolds are also invariant, for example, the main diagonal intersection manifold $M_{i j k}^{ \pm}\left(d_{s}\right)=M_{i j}^{+} \cap M_{j k}^{+} \cap M_{i k}^{+} \cap M_{i j}^{-} \cap M_{j k}^{-} \cap$ $M_{i k}^{-}$.

(2) There exists an intersection manifold $M_{s}^{*}(d)$ with the number of clusters

$$
d= \begin{cases}\frac{(n+1)\left(n^{2}+n+2\right)}{2}, & \text { for } N=2 n+1 \\ \frac{n\left(n^{2}-n+2\right)}{2}, & \text { for } N=2 n,\end{cases}
$$


being a particular case of the intersection manifold $M_{s}^{\times}\left(n_{1}, n_{2}, n_{3}\right)$.

The invariant manifold $M_{s}^{*}(d)=M_{i, j, k}^{ \pm}\left(d_{s}\right) \cap$ $M_{s}^{\times}\left(n_{1}, n_{2}, n_{3}\right)$ defines simultaneously the symmetries of synchronized oscillators with respect to all the diagonals sections of the 3 -D lattice and to the middle of the rows, columns and verticals of the lattice.

In the following we will present the stable cluster regimes defined by the cluster synchronization manifold $M_{s}^{*}(d)$ for a 3-D lattice of coupled chaotic Rössler oscillators.

We have examined the existence of main cluster synchronization manifolds and we shall now consider the existence of cluster synchronization manifolds of the 2-D planes of the 3-D lattice (1) and provide this consideration with examples for concrete numbers.

\subsection{Two-dimensional lattice}

In this case we reduce the system (2) to the following form

$$
\dot{X}=F(X)+\varepsilon_{1} P \triangle_{i}+\varepsilon_{2} P \triangle_{j},
$$

with zero-flux or periodic BC. All notations are similar to those of the system (2).

\subsubsection{Rectangular $N_{1} \times N_{2}$ lattice}

Applying Proposition 2.1 to the 2-D lattice (3), we obtain the existence of the following cluster synchronization manifolds.

(1) There exists a symmetrical invariant manifold $M_{s}^{r}\left(n_{1}, N_{2}\right)$ with $n_{1}=\operatorname{int}\left(\left(N_{1}+1\right) / 2\right)$ defined by the equalities $\left\{X_{N_{1}-i+1, j}=X_{i, j}=U_{i, j}\right.$, $\left.i=1,2, \ldots, \operatorname{int}\left(\left(N_{1}+1\right) / 2\right), j=1,2, \ldots, N_{2}\right\}$.

(2) There exists an asymmetrical invariant manifold $M_{a}^{r}\left(n_{1}, N_{2}\right)$, where $N_{1}=p_{1} \cdot n_{1}\left(p_{1}\right.$ and $n_{1}$ are arbitrary integers), defined by the equalities $\left\{X_{i, j}=X_{i+2 n_{1} l, j}\right.$, $l=1,2, \ldots, \operatorname{int}\left(\left(p_{1}-1\right) / 2\right)$ and $X_{i, j}=$ $X_{-i+1+2 n_{1} l, j}, \quad l=1,2, \ldots, \operatorname{int}\left(p_{1} / 2\right), \quad i=$ $\left.1,2, \ldots, n_{1}, j=1,2, \ldots, N_{2}\right\}$.

(3) The system (3) has similar invariant manifolds defining cluster synchronization in the $j$ th lattice direction.

Thus, in the case of the 2-D lattice, horizontal and vertical lines of the lattice play the roles of separate oscillators forming the clusters in the 1-D array.

Similar to the 3-D lattice case, there exists a family of intersection manifolds $M_{s, a}^{\times}\left(n_{1}, n_{2}\right)=$ $M_{s, a}^{r}\left(n_{1}, N_{2}\right) \cap M_{s, a}^{c}\left(N_{1}, n_{2}\right)$, being an intersection of any row and any column synchronization manifolds existing in the case of the 1-D chain.

Example 1. Let us consider a 2-D lattice containing $N_{1}=3$ and $N_{2}=5$ oscillators in the two lattice directions and let us use natural numbers as the symbols $a_{i, j}$ standing in the matrix $A$ such that the oscillators, denoted by the same digit, have identical dynamics. We present main cluster synchronization manifolds which exist for these $N_{1}$ and $N_{2}$. The symbolic representations of the manifolds $M_{s}^{r}(1,5)$ and $M_{s}^{r}(2,5)$ have the following forms

$$
\left(\begin{array}{lllll}
1 & 2 & 3 & 4 & 5 \\
1 & 2 & 3 & 4 & 5 \\
1 & 2 & 3 & 4 & 5
\end{array}\right) \text { and }\left(\begin{array}{lllll}
1 & 3 & 5 & 7 & 9 \\
2 & 4 & 6 & 8 & 10 \\
1 & 3 & 5 & 7 & 9
\end{array}\right)
$$

respectively. The manifold $M_{s}^{r}(1,5)$ corresponds to full synchronization of the rows of the lattice and desynchronization of the columns, and the manifold $M_{s}^{r}(2,5)$ defines two-cluster synchronization of the rows and a desynchronization pattern between the columns.

The symbolic representations of the intersection synchronization manifolds $M_{s}^{\times}(1,3)$ and $M_{s}^{\times}(2,3)$, also existing in this case, attain the forms,

$$
\left(\begin{array}{lllll}
1 & 2 & 3 & 2 & 1 \\
1 & 2 & 3 & 2 & 1 \\
1 & 2 & 3 & 2 & 1
\end{array}\right) \text { and }\left(\begin{array}{lllll}
1 & 3 & 5 & 3 & 1 \\
2 & 4 & 6 & 4 & 2 \\
1 & 3 & 5 & 3 & 1
\end{array}\right)
$$

respectively. The manifold $M_{s}^{\times}(1,3)$ defines full synchronization of the rows and cluster synchronization between the columns, where $1 \times 3=3$ is the number of clusters, and the manifold $M_{s}^{\times}(3,2)$ determines cluster synchronization both in the rows and columns and the number of clusters is $2 \times 3=6$.

Example 2. Let $N_{1}=3$ and $N_{2}=6$. The main intersection invariant manifolds $M_{s}^{\times}(2,3)$ and $M_{a}^{\times}(2,2)$ have the following forms

$$
\left(\begin{array}{llllll}
1 & 2 & 3 & 3 & 2 & 1 \\
4 & 5 & 6 & 6 & 5 & 4 \\
1 & 2 & 3 & 3 & 2 & 1
\end{array}\right)
$$


and

$$
\left(\begin{array}{llllll}
1 & 2 & 2 & 1 & 1 & 2 \\
3 & 4 & 4 & 3 & 3 & 4 \\
1 & 2 & 2 & 1 & 1 & 2
\end{array}\right)
$$

respectively.

Let us now consider the system (3) under the additional conditions on the function $F(X)$ to be odd: $F(-X)=-F(X)$. Here, the system (3) is centrally symmetric with respect to the zero point $X=0$ that is an equilibrium point of the system (3). In this case, the 1-D array of diffusively coupled systems (1) for $\varepsilon_{1}=0$ (or $\varepsilon_{2}=0$ ) has invariant manifolds, defining the existence of antiphase synchronization. Such antiphase synchronization is observed, for example, in a system of two coupled oscillators where all corresponding variables of the two individual oscillators are equal with opposite sign. For $\varepsilon_{1} \neq 0\left(\varepsilon_{2} \neq 0\right)$ we have the straightforward transfer of the appropriate statements to the existence of row (column) antiphase synchronization manifolds of the 2-D lattice.

Proposition 2.5. Under the condition $F(-X)=$ $-F(X)$ :

(1) the system (3) has a row manifold $M_{t}^{r}\left(n_{1}, N_{2}\right)$ with $n_{1}=\operatorname{int}\left(\left(N_{1}+1\right) / 2\right)$ defined by the equalities $\left\{-X_{N_{1}-i+1, j}=X_{i, j}=U_{i, j}, i=\right.$ $\left.1,2, \ldots, n_{1}, j=1,2, \ldots, N_{2}\right\}$ for even $N_{1}=2 n_{1}$ and by the equalities $\left\{-X_{N_{1}-i+1, j}=\right.$ $X_{i, j}=U_{i, j}, i=1,2, \ldots, n_{1}, X_{n_{1}+1, j} \equiv 0$, $\left.j=1,2, \ldots, N_{2}\right\}$ for odd $N_{1}=2 n_{1}+1$.

(2) The system (3) has the similar column manifold $M_{t}^{c}\left(N_{1}, n_{2}\right)$ with $n_{2}=\operatorname{int}\left(\left(N_{2}+1\right) / 2\right)$.

Corollary. Obviously, the same statements on the existence of antiphase synchronization manifolds may be made with respect to the 2- $D$ planes $P^{l}$ of the 3-D lattice (2) having the odd function $F(X)$.

Example 3. Let us use positive numbers for inphase synchronization and negative ones for antiphase synchronization as the symbols $a_{i, j}$ in the matrix $A$, " 0 " denotes $X_{i, j}=0$. Consider two 2-D lattices with $N_{1}=3, N_{2}=4$ and $N_{1}=3$, $N_{2}=5$. The manifolds $M_{t}^{c}(3,2)$ and $M_{t}^{c}(3,3)$, existing due to Proposition 2.6, have the symbolic representations

$$
\left(\begin{array}{llll}
1 & 4 & -4 & -1 \\
2 & 5 & -5 & -2 \\
3 & 6 & -6 & -3
\end{array}\right) \text { and }\left(\begin{array}{ccccc}
1 & 4 & 0 & -4 & -1 \\
2 & 5 & 0 & -5 & -2 \\
3 & 6 & 0 & -6 & -3
\end{array}\right)
$$

respectively.

\subsubsection{Square $N \times N$ lattice}

Let us consider now a particular case of the system (3) with $N_{1}=N_{2}=N$ and $\varepsilon_{1}=\varepsilon_{2}=\varepsilon$.

By reference to Proposition 2.5, we state that the spatiotemporal dynamics of the 2-D square lattice exhibits cluster synchronization regimes defined by the existence of the invariant manifolds $M^{+}(d)$ and $M^{-}(d)$, where $d=N(N+1) / 2$ is the number of clusters. The manifold $M^{+}(d)$ is defined by the equalities $\left\{X_{j, i}=X_{i, j}=U_{i, j}, i=\overline{1, N}\right.$, $i \leq j \leq N\}$, and $M^{-}(d)$ is defined by the equalities $\left\{X_{N-j+1, N-i+1}=X_{i, j}=U_{i, j}, i=\overline{1, N}\right.$, $1 \leq j \leq N-i+1\}$.

The dynamics in the cluster manifolds $M^{+}(d)$ and $M^{-}(d)$ defines simple symmetries of the synchronized oscillators with respect to the principal and secondary diagonals of the 2-D lattice, respectively. Obviously, the intersection symmetrical manifold $M^{ \pm}\left(d_{s}\right)=M^{+}(d) \cap M^{-}(d)$, where

$$
d_{s}= \begin{cases}(n+1)^{2}, & \text { for odd } N=2 n+1 \\ n(n+1), & \text { for even } N=2 n\end{cases}
$$

is also invariant.

Similar to the 3-D lattice case, there also exists the intersection manifold $M_{s}^{*}(d)$ with the number of clusters

$$
d= \begin{cases}\frac{(n+1)(n+2)}{2}, & \text { for } N=2 n+1 \\ \frac{n(n+1)}{2}, & \text { for } N=2 n .\end{cases}
$$

The invariant manifold $M_{s}^{*}(d)=M^{ \pm}\left(d_{s}\right) \cap$ $M_{s}^{\times}\left(n_{1}, n_{2}\right)$ defines simultaneously the symmetries of synchronized oscillators with respect to the two diagonals and to the middles of the rows and columns of the lattice.

Example 4. Let the square lattice (3) be composed from $N=5$ and $\varepsilon_{1}=\varepsilon_{2}=\varepsilon$. For this case we present the invariant manifolds $M^{+}(15), M^{ \pm}(9)$, 
and $M_{s}^{*}(6)$

$$
\begin{gathered}
\left(\begin{array}{rrrrr}
1 & 2 & 3 & 4 & 5 \\
2 & 6 & 7 & 8 & 9 \\
3 & 7 & 10 & 11 & 12 \\
4 & 8 & 11 & 13 & 14 \\
5 & 9 & 12 & 14 & 15
\end{array}\right)\left(\begin{array}{l}
1 \\
2 \\
3 \\
4 \\
5
\end{array}\right. \\
\times\left(\begin{array}{rrrrr}
1 & 2 & 3 & 2 & 1 \\
2 & 4 & 5 & 4 & 2 \\
3 & 5 & 6 & 5 & 3 \\
2 & 4 & 5 & 4 & 2 \\
1 & 2 & 3 & 2 & 1
\end{array}\right)
\end{gathered}
$$$$
\left.\begin{array}{llll}
2 & 3 & 4 & 5 \\
6 & 7 & 8 & 4 \\
7 & 9 & 7 & 3 \\
8 & 7 & 6 & 2 \\
4 & 3 & 2 & 1
\end{array}\right)
$$

respectively.

Let us study now the embedding of the invariant synchronization manifolds and thus define possible routes of transition from completely unsynchronized pattern to a single spatially homogeneous cluster defining full synchronization with increasing coupling between oscillators.

First we consider the $N \times N$ lattice (3) with $\varepsilon_{1}=\varepsilon_{2}=\varepsilon$ and with a prime number $N=2 n+1$. Below we list linear inphase synchronization manifolds existing in the case of zero-flux BC.

(1) $M^{+}(d), M^{-}(d)$, and $M^{ \pm}\left(d_{s}\right)$ defining the symmetries of synchronized oscillators of the square lattice with respect to the principal and secondary diagonals of the lattice.

(2) $M^{*} \equiv M_{s}^{*}((n+1)(n+2) / 2)$.

(3) $M^{\mathrm{des} / \mathrm{cl}} \equiv M_{s}^{c}(N, n+1)=\left[\left\{r_{1}, \ldots, r_{N}\right\} \times\right.$ $\left.\left\{c_{1}, \ldots, c_{n+1}, \ldots, c_{1}\right\}\right]$ defining the $(n+1)$ cluster synchronization of the vertical columns and desynchronization between the horizontal rows.

(4) $M^{\text {des } / \text { syn }} \equiv M^{c}(N, 1)=\left[\left\{r_{1}, \ldots, r_{N}\right\} \times\right.$ $\left.\left\{c_{1}, c_{1}, c_{1}, \ldots\right\}\right]$.

(5) $M^{\mathrm{cl} / \mathrm{cl}} \equiv M_{s}^{\times}(n+1, n+1)=\left[\left\{r_{1}, \ldots\right.\right.$, $\left.\left.r_{n+1}, \ldots, r_{1}\right\} \times\left\{c_{1}, \ldots, c_{n+1}, \ldots, c_{1}\right\}\right]$ defining two different $(n+1)$-cluster synchronization regimes of the horizontal and vertical lines of the lattice.

(6) $M^{\mathrm{cl} / \mathrm{syn}} \equiv M_{s}^{c}(n+1,1)=\left[\left\{r_{1}, \ldots, r_{n+1}, \ldots\right.\right.$, $\left.\left.r_{1}\right\} \times\left\{c_{1}, \ldots, c_{1}, \ldots, c_{1}\right\}\right]$ corresponding to full synchronization of the vertical columns and $(n+1)$-cluster synchronization of the horizontal rows.

(7) $M(1) \equiv\left[\left\{r_{1}, \ldots, r_{1}, \ldots, r_{1}\right\} \times\left\{c_{1}, \ldots\right.\right.$, $\left.\left.c_{1}, \ldots, c_{1}\right\}\right]$ determining synchronization of all oscillators of the lattice.
These manifolds are embedded as follows

$$
\begin{array}{cl}
\supset M^{\text {des/syn }} & \supset M^{\mathrm{cl} / \mathrm{syn}} \supset M(1) . \\
M^{\text {des } / \mathrm{cl}} & \cap \\
\supset M^{\mathrm{cl} / \mathrm{cl}} \supset & M^{*} \\
& \cap \\
& M^{ \pm} \\
& \cap \\
& M^{+} M^{-}
\end{array}
$$

Remark. Obviously, the vertical and horizontal lines of the 2-D lattice may be inverted and the same clusters and the embedding may be rewritten with respect to the horizontal rows.

Consider the $N \times N$ lattice (3) with $N=p \cdot n_{1}$, where $p$ and $n_{1}$ are arbitrary integers greater than 1. By reference to Proposition 2.1 that is applied for the 2-D lattice case, we note that the system (3) has the additional asymmetrical invariant manifolds $M_{a}^{r}\left(n_{1}, N\right)$ and $M_{a}^{c}\left(N, n_{1}\right)$. Therefore, the collection of possible modes of cluster synchronization in the 2-D lattice is even richer and additional intersection invariant manifolds may be easily obtained as the topological product of the cluster patterns, and the embedding (4) is extendable.

These embeddings may determine the order of appearance of cluster synchronization regimes with changing coupling between the individual oscillators of the lattice (3). Thus after having considered the existence of embedded linear invariant manifolds, the main problem is to study their stability and the order of stabilization for concrete coupled chaotic oscillators. That will be the topic of the following sections.

\section{Stability of Invariant Manifolds}

The objective of this section is to obtain conditions of global asymptotic stability of invariant manifolds corresponding to different synchronization regimes in the volume lattice (1) with zero-flux BC and the main question in this study is under which conditions on the coupling strengths $\varepsilon_{1}, \varepsilon_{2}$ and $\varepsilon_{3}$, the numbers of oscillators $N_{1}, N_{2}$ and $N_{3}$, and parameters of the individual oscillators full synchronization of all spatial sites arises. 


\subsection{Sufficient conditions of global stability of synchronization in the 3-D lattice}

Let us study first global stability of the manifold $M^{v}\left(N_{1}, N_{2}, 1\right)$ defining synchronization between 2 -D lattices in the $k$ th direction of the volume lattice (1).

Using the notation

$$
\Phi_{i, j, k}=x_{i, j, k}-x_{i, j, k+1}, \quad \Psi_{i, j, k}=y_{i, j, k}-y_{i, j, k+1},
$$

we derive the difference equations from the general system (1) with zero-flux BC

$$
\begin{aligned}
\dot{\Phi}_{i, j, k}= & A(t) \cdot \Phi_{i, j, k}+(\alpha(t))^{T} \Psi_{i, j, k}+\varepsilon_{1} \Delta_{i} \\
& +\varepsilon_{2} \Delta_{j}+\varepsilon_{3} \Delta_{k}, \\
\dot{\Psi}_{i, j, k}= & B(t) \cdot \Phi_{i, j, k}+\Lambda(t) \cdot \Psi_{i, j, k}, \quad i=1,2, \ldots, N_{1}, \\
& j=1,2, \ldots, N_{2}, \quad k=1,2, \ldots, N_{3}-1
\end{aligned}
$$

with BC $\Phi_{0, j, k}=\Phi_{1, j, k}, \Phi_{i, 0, k}=\Phi_{i, 1, k}, \Phi_{N_{1}+1, j, k}=$ $\Phi_{N_{1}, j, k}, \Phi_{i, N_{2}+1, k}=\Phi_{i, N_{2}, k}, \Phi_{i, j, 0}=\Phi_{i, j, N_{3}}=0$. Here, $A(t)=f_{x}^{\prime}\left(x^{*}\right)$ is a scalar function, $(\alpha(t))^{T}=$ $f_{y}^{\prime}\left(y^{*}\right)$ and $B(t)=g_{x}^{\prime}\left(x^{*}\right)$ are $(m-1)$ - column vector functions, $\Lambda(t)=g_{y}^{\prime}\left(y^{*}\right)$ is an $(m-1) \times(m-1)$ Jacobian matrix, $x^{*}(t) \in\left[x_{i, j, k}, x_{i, j, k+1}\right]$ and $y^{*}(t) \in$ $\left[y_{i, j, k}, y_{i, j, k+1}\right]$ are the values coming from the Lagrange mean-value theorem and they are time dependent via the solutions of Eq. (5).

We introduce the auxiliary system

$$
\begin{aligned}
& \dot{\Phi}=-a \Phi+(\alpha(t))^{T} \Psi, \\
& \dot{\Psi}=c(t) \Phi+B(t) \Psi,
\end{aligned}
$$

where $a=$ const $>0$.
This system is identical to the individual subsystem of the system (5) except that $A(t)$ is changed to $-a$.

Similar to [Belykh et al., 1993; Belykh et al., 2000], the following assertion holds.

Theorem 3.1. (Sufficient conditions of synchronization of 2-D lattices in the kth direction). Assume that there exists a positive definite Lyapunov function

$$
V(\Phi, \Psi)=\frac{\left(\Phi^{2}+\Psi^{T} H \Psi\right)}{2}
$$

with some symmetrical matrix $H$ such that the derivative

$$
\dot{V}(\Phi, \Psi)=-a \Phi^{2}+\frac{d}{d t}\left(\Psi^{T} H \Psi\right)+\Phi \alpha^{T} \Psi
$$

with respect to the system (6) is negative definite for any function $\alpha(t), c(t), B(t)$ that is generated by solutions of Eq. (5).

Then the manifold $M^{v}\left(N_{1}, N_{2}, 1\right)$ is globally asymptotically stable for

$$
\begin{gathered}
0<\nu_{3}<1 \quad \text { and } \quad N_{3} \leq \operatorname{Int}\left(\frac{\pi}{\arccos \nu_{3}}\right) ; \\
\nu_{3} \geq 1 \quad \text { for any } \quad N_{3}>1,
\end{gathered}
$$

where $\nu_{3}=1-(a-b) / 2 \varepsilon_{3}$ and $b \equiv \max _{x \in R^{m}} A(t)$.

Proof. Consider the function

$$
W=\sum_{i=1}^{N_{1}} \sum_{j=1}^{N_{2}}\left\{\sum_{k=1}^{N_{3}-1} \frac{\left(\Phi_{i, j, k}^{2}+\Psi_{i, j, k}^{T} H \Psi_{i, j, k}\right)}{2}\right\} .
$$

Calculating its time derivative along the trajectories of Eq. (5), we obtain

$$
\begin{aligned}
\dot{W}= & \sum_{i=1}^{N_{1}} \sum_{j=1}^{N_{2}} \sum_{k=1}^{N_{3}-1}\left\{A(t) \Phi_{i, j, k}^{2}+\Phi_{i, j, k} \alpha^{T} \Psi_{i, j, k}+d\left(\Psi_{i, j, k}^{T} H \Psi_{i, j, k}\right) / d t+\varepsilon_{1} \Phi_{i, j, k}\left(\Phi_{i-1, j, k}-2 \Phi_{i, j, k}+\Phi_{i+1, j, k}\right)\right. \\
& \left.+\varepsilon_{2} \Phi_{i, j, k}\left(\Phi_{i, j-1, k}-2 \Phi_{i, j, k}+\Phi_{i, j+1, k}\right)+\varepsilon_{3} \Phi_{i, j, k}\left(\Phi_{i, j, k-1}-2 \Phi_{i, j, k}+\Phi_{i, j, k+1}\right)\right\} .
\end{aligned}
$$

Taking into account the equality (8), we can transform Eq. (11) to the following form

$$
\begin{aligned}
\dot{W}= & \sum_{i=1}^{N_{1}} \sum_{j=1}^{N_{2}} \sum_{k=1}^{N_{3}-1}\left\{A(t) \Phi_{i, j, k}^{2}+a \Phi_{i, j, k}^{2}+\varepsilon_{3} \Phi_{i, j, k}\left(\Phi_{i, j, k-1}-2 \Phi_{i, j, k}+\Phi_{i, j, k+1}\right)\right\} \\
& +\sum_{i=1}^{N_{1}} \sum_{j=1}^{N_{2}} \sum_{k=1}^{N_{3}-1} \dot{V}\left(\Phi_{i, j, k}, \Psi_{i, j, k}\right)+\sum_{j=1}^{N_{2}} \sum_{k=1}^{N_{3}-1}\left\{\varepsilon_{1} \sum_{i=1}^{N_{1}} \Phi_{i, j, k}\left(\Phi_{i-1, j, k}-2 \Phi_{i, j, k}+\Phi_{i+1, j, k}\right)\right\} \\
& +\sum_{i=1}^{N_{1}} \sum_{k=1}^{N_{3}-1}\left\{\varepsilon_{2} \sum_{j=1}^{N_{2}} \Phi_{i, j, k}\left(\Phi_{i, j-1, k}-2 \Phi_{i, j, k}+\Phi_{i, j+1, k}\right)\right\} .
\end{aligned}
$$


Let us now study the properties of three last terms of the sum (12).

(1) $\sum_{i=1}^{N_{1}} \sum_{j=1}^{N_{2}} \sum_{k=1}^{N_{3}-1} \dot{V}\left(\Phi_{i, j, k}, \Psi_{i, j, k}\right)<0$, since the time derivative $\dot{V}$ of each individual auxiliary system is negative definite, due to the as(2) sumption of Theorem 3.1.

$$
\begin{aligned}
S_{j, k}^{(1)} & \equiv \sum_{i=1}^{N_{1}}\left\{\Phi_{i, j, k}\left(\Phi_{i-1, j, k}-2 \Phi_{i, j, k}+\Phi_{i+1, j, k}\right)\right\} \\
& =\sum_{i=1}^{N_{1}-1}\left\{-\left(\Phi_{i+1, j, k}-\Phi_{i, j, k}\right)^{2}\right\} \leq 0, \\
S_{i, k}^{(2)} & \equiv-\sum_{j=1}^{N_{2}-1}\left\{-\left(\Phi_{i, j+1, k}-\Phi_{i, j, k}\right)^{2}\right\} \leq 0,
\end{aligned}
$$

then the two last terms of Eq. (12) $\sum_{j=1}^{N_{2}}$. $\sum_{k=1}^{N_{3}-1}\left\{\varepsilon_{1} S_{j, k}^{(1)}\right\}$ and $\sum_{i=1}^{N_{1}} \sum_{k=1}^{N_{3}-1}\left\{\varepsilon_{2} S_{i, k}^{(2)}\right\}$ are also negative definite.

Thus, the derivative $\dot{W}$ along the trajectories of Eq. (5) is negative definite if the quadratic form

$$
\begin{aligned}
Q= & \sum_{k=1}^{N_{3}-1}\left\{A(t) \Phi_{i, j, k}^{2}+a \Phi_{i, j, k}^{2}\right. \\
& +\varepsilon_{3} \Phi_{i, j, k}\left(\Phi_{i, j, k-1}-2 \Phi_{i, j, k}+\Phi_{i, j, k+1}\right\}
\end{aligned}
$$

with $\Phi_{i, j, N_{3}}=0$, is in turn negative definite, while is true under the condition (9).

The parameter $a>0$, which replaces $f_{x}^{\prime}$ that may change sign, is the minimal damping of variable $x$ needed to make the auxiliary system (6) globally asymptotically stable.

Let us study now the stability condition (9). If $\nu \geq 1$, then $b<0$, and the system (5) is globally asymptotically stable such that the trivial behavior of individual oscillators of the lattice is defined by stable equilibria.

If $0<\nu<1$, then $b \equiv \max _{x \in \text { attractor }} f_{x}^{\prime}>0$, the oscillators of the lattice may exhibit complicated individual dynamics, and the inequality $\varepsilon_{3}>$ $(a+b) / 2$ holds. The stability conditions (9) have another limitation for fixed $N_{3}: N_{3} \leq \operatorname{Int}\left(\pi / \arccos \nu_{3}\right)$. Thus we obtain the following sufficient condition on coupling and the individual oscillator system for global stability of the synchronization manifold
$M^{v}\left(N_{1}, N_{2}, 1\right)$ :

$$
\varepsilon_{3}>\frac{(a+b)}{4 \sin ^{2}\left(\frac{\pi}{2 N_{3}}\right)} .
$$

Note that similar sufficient conditions of synchronization of 2-D lattices may be written for the three lattice directions as follows

$$
\varepsilon_{m}>\varepsilon_{m}^{\mathrm{suf}}=\frac{(a+b)}{4 \sin ^{2}\left(\frac{\pi}{2 N_{m}}\right)}, \quad m=1,2,3 .
$$

Proposition 3.1. (Sufficient conditions of full synchronization). If $N_{1}>N_{2}>N_{3}$, then

$$
\varepsilon_{1}^{\text {suf }}>\varepsilon_{2}^{\text {suf }}>\varepsilon_{3}^{\text {suf }}
$$

and full synchronization of all oscillators of the volume lattice may be achieved via the changing coupling parameters along the "diagonal" $\varepsilon=\varepsilon_{1}=$ $\varepsilon_{2}=\varepsilon_{3}$ in the $\left(\varepsilon_{1}, \varepsilon_{2}, \varepsilon_{3}\right)$ parameter space.

The intersection of the three 2-D lattices of synchronized oscillators leads first to synchronization between the 2-D lattices in the $k$ th direction, then to synchronization between the lines of synchronized oscillators in the $j$ th direction within the $2-\mathrm{D}$ synchronized lattices and finally to synchronization between oscillators within the synchronized lines.

It turned out that the sufficient conditions for full synchronization in three lattice directions of the 3 -D lattice are similar to those obtained for a 1-D chain of coupled Chua circuits [Belykh et al., 1993].

It is interesting to note that independently of the parameters $a$ and $b$ related to the stability properties of the individual oscillator, we have the following equality for each pair of $\left(\varepsilon_{1}^{\text {suf }}, N_{1}\right),\left(\varepsilon_{2}^{\text {suf }}, N_{2}\right)$ and $\left(\varepsilon_{3}^{\text {suf }}, N_{3}\right)$ :

$$
\varepsilon_{m}^{\mathrm{suf}} \cdot \sin ^{2}\left(\frac{\pi}{2 N_{m}}\right)=\frac{(a+b)}{4}=C_{1},
$$

where $C_{1}$ is a constant.

This led us to a natural estimate of the relation between necessary and sufficient conditions for coupling needed for full global synchronization.

\subsection{Estimation of necessary conditions of full synchronization}

Let us study first the expression (15). For $N \gg 1$, $\sin (\pi / 2 N) \cong \pi / 2 N$, and the formula (15) may be 
rewritten in the following form

$$
\varepsilon_{m}^{\text {suf }}=C_{2} N_{m}^{2}
$$

where $C_{2}$ is a constant that is defined by the properties of the individual oscillator.

Then the following question arises: if the dependence of the sufficient synchronization coupling values on the number of oscillators has a quadratic law, then which is the dependence between the real threshold coupling values providing the necessary conditions under which global synchronization arises in a lattice?

Answering this question, we come to a conjecture on the estimation of necessary conditions for full synchronization in a $3-\mathrm{D}$ lattice of diffusively coupled oscillators.

Conjecture. If the 3-D lattice system (1) satisfies the variational equations (5) and the conditions of Theorem 3.1, i.e. if full global synchronization arises in the system with increasing the coupling, then the formula

$$
\varepsilon_{m}^{\text {nec }}=C N_{m}^{2}, \quad m=1,2,3, \text { and } N_{m}>3
$$

is a good estimate of the necessary conditions of full synchronization of oscillators in the 3-D lattice and presents a simple quadratic law of the dependence of necessary threshold coupling values $\varepsilon_{m}^{\text {nec }}$ on the number of oscillators $N_{m}$ in each lattice direction. Here, $C$ is a new constant which may be considered, with some accuracy, as an invariant of the coupled system.

Thus, we conjecture that if we find a synchronization threshold value $\varepsilon_{m}^{\text {nec }}\left(N_{m}\right)$ for $m=1,2,3$ (say, from numerical simulations) providing the necessary condition of synchronization for one particular $N_{m}>3$ in one lattice direction of a 3-D lattice of coupled oscillators satisfying the conditions of Theorem 3.1, we may once calculate the constant $C$ from Eq. (16) and thus to predict the relation between $N_{m}$ and $\varepsilon_{m}^{\text {nec }}\left(N_{m}\right)$ for any arbitrary $N_{m}>3$ and each lattice direction. This estimation is a natural corollary of Theorem 3.1 for the 3-D lattices and is also connected with the $\mathrm{Wu}-\mathrm{Chua}$ conjecture.

$\mathrm{Wu}$ and Chua [1996] conjectured that there is the exact relation between the coupling constants required for the onset of global synchronization and the eigenvalues of coupling matrices in linearly coupled arrays of oscillators. The conjecture is the following. Suppose that (i) two similar 1-D arrays are composed of $N_{1}$ and $N_{2}$ oscillators, respectively, (ii) $\varepsilon_{N_{1}}^{\text {nec }}$ and $\varepsilon_{N_{2}}^{\text {nec }}$ are the coupling constants under which global synchronization of the oscillators arises in the two arrays, respectively, (iii) $\mu_{1}\left(N_{1}\right)$ and $\mu_{2}\left(N_{2}\right)$ are the least negative eigenvalues of the coupling matrices, respectively. Suppose that the following equality holds

$$
\varepsilon_{N_{1}}^{\mathrm{nec}} \mu_{1}=\varepsilon_{N_{2}}^{\mathrm{nec}} \mu_{2} .
$$

Then the array with $N_{2}$ oscillators globally synchronizes if and only if the array with $N_{1}$ globally synchronize.

Recently, it was shown [Pecora, 1998; Pogromsky \& Nijmeijer, 2001] that the $\mathrm{Wu}-\mathrm{Chua}$ conjecture is false in general, for example, for chaotic arrays which have desynchronization bifurcations and lose the synchronization regime with an increase of coupling. A 1-D array of $x$-coupled Rössler systems belongs to this class and exhibits so-called shortwavelength bifurcations corresponding to desynchronization [Heagy et al., 1994]. A mechanism of desynchronization which has direct relation to these bifurcations was proposed [Belykh et al., 2000]. Usually, coupled chaotic systems have no sharp synchronization threshold due to the existence of attractor bubbling [Ashwin et al., 1994a; 1994b; Hasler \& Maistrenko, 1997] and riddled basins of attraction [Alexander et al., 1992]. Therefore the exact coupling value for the synchronization threshold cannot be nearly defined, and therefore the relation between the necessary conditions on coupling for different numbers of oscillators may only be roughly predicted.

Let us digress for a moment from the study of the 3-D lattice of coupled oscillators and check the estimation formula (16) for 1-D arrays of coupled oscillators.

We study first a 1-D array of diffusively $x$-coupled Lorenz systems with zero-flux BC. We found numerically the threshold synchronization values of coupling $\varepsilon^{\text {nec }}$ for different numbers of oscillators $N$. Then we calculated $C$ from the formula $C=\varepsilon^{\text {nec }} / N^{2}$ for different $N$ [see Fig. 1(a)].

As the second example of 1-D arrays of coupled chaotic systems admitting the onset of global synchronization, we consider a 1 -D array of $x$-coupled generalized Chua circuits with zero-flux BC. As for the coupled Lorenz systems, we calculated the constant $C$ for different $N$ [see Fig. 1(b)].

It appeared that our conjecture is valid for these two cases, and for $N>3$, the constants $C$ may be considered, with some accuracy, as invariants of these two arrays of coupled systems. Obviously, the 


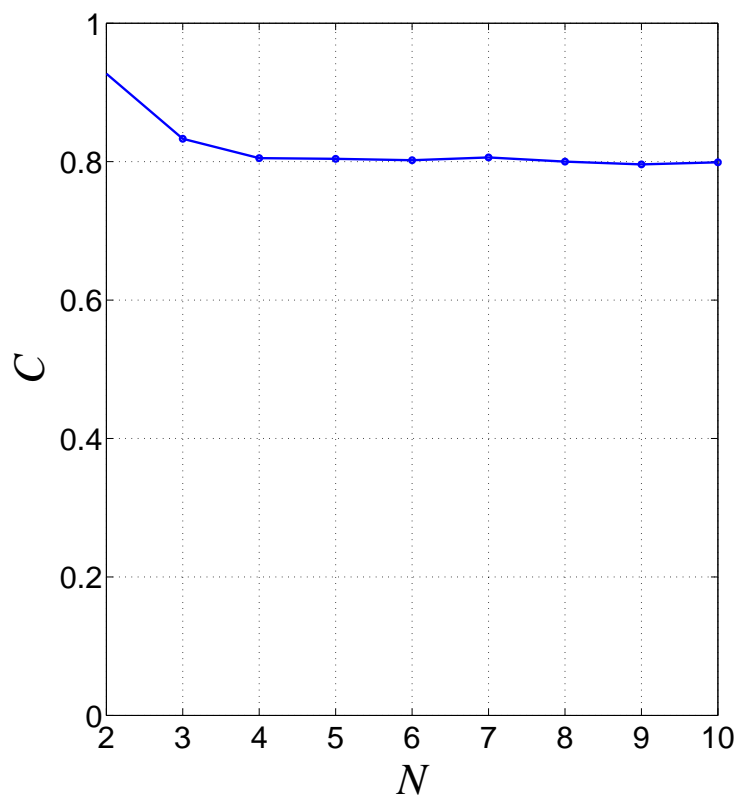

(a)

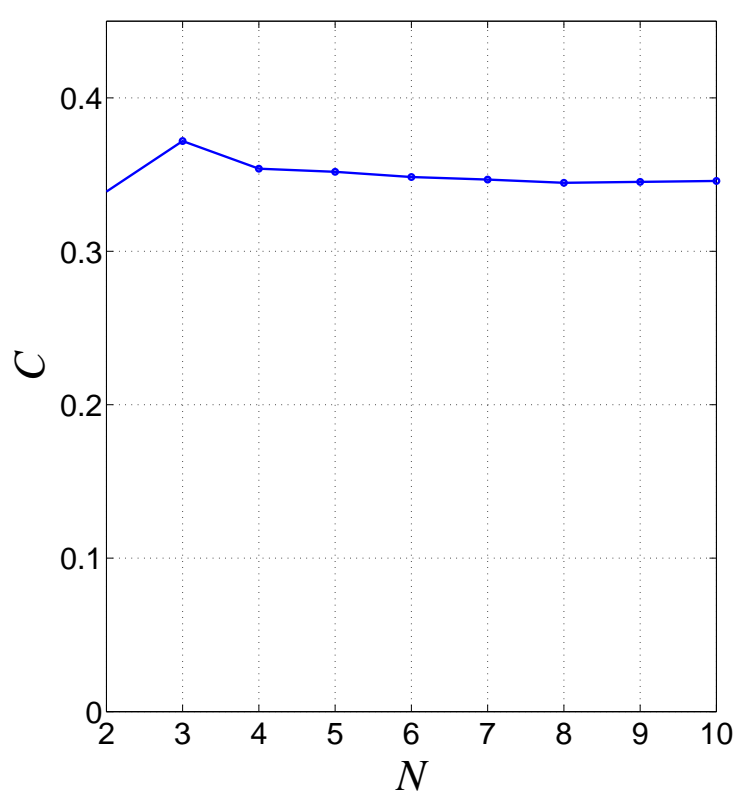

(b)

Fig. 1. Dependence of the constant $C$ on the number of oscillators $N$ in a 1-D array of coupled chaotic systems with zero-flux BC. The threshold synchronization values $\varepsilon^{\text {nec }}$ are found numerically for each $N(N=2, \ldots, 10)$ and the constant $C$ is calculated from Eq. (16). (a) $x$-coupled Lorenz systems $(r=27, b=8 / 3, \sigma=10)$. (b) $x$-coupled generalized Chua circuits (24) $\left(\alpha=10.5, b_{1}=-110.17, b_{2}=10.5, a_{11}=-3.8, a_{12}=-42, a_{21}=0.36, a_{22}=2.78\right)$.

formula (16) is valid for 3 -D lattices of $x$-coupled Lorenz systems and Chua circuits. Therefore synchronization in any lattice direction for arbitrary $N_{m}$ may be predicted by knowing a single value of synchronization threshold for one particular $N>3$. Remark that the estimation formula (16) is asymptotic and becomes more effective when the number of oscillators increases.

Note that the $\mathrm{Wu}-\mathrm{Chua}$ conjecture, while holding only approximately, is also useful for these particular cases. We have obtained our estimation formula independently and it appeared that in some context, we have corrected the $\mathrm{Wu}-\mathrm{Chua}$ conjecture for a 1-D array of diffusively coupled systems admitting the onset of global (arising from all initial conditions) synchronization with increasing coupling and we have suggested the estimation formula that is also valid for 3-D lattices. Thus we have conjectured that the synchronization threshold in such arrays composed from an arbitrary number $N>3$ of oscillators may be well predicted from synchronization in four coupled cells.

We note that lattices of most diffusively coupled systems belong to the class of dynamical systems satisfying the conditions of Theorem 3.1, such that the estimation formula (16) can be applied.
Obviously, the formula (16) is not valid for all dynamical systems that are diffusively coupled in a lattice. For example, it does not handle a lattice of coupled Rössler oscillators, where the synchronization regime loses its global stability with increasing coupling between the oscillators.

In the next sections, we will study two cases of 2-D and 3-D lattices of coupled Lur'e systems and coupled Rössler oscillators presenting the two different synchronization scenarios.

\section{Coupled Lur'e Systems}

We start the study of 3-D lattices of coupled concrete systems by considering the system (1) with zero-flux BC and a Lur'e system as the individual oscillator of the lattice. The individual dynamics of each oscillator of the lattice is described by the following dynamical system

$$
\left\{\begin{array}{l}
\dot{x}=f(x)+y+z \\
\dot{y}=b_{1} f(x)-\lambda y-\alpha z \\
\dot{z}=b_{2} f(x)+\omega y-\lambda z,
\end{array}\right.
$$

where $\lambda, \alpha, \omega, b_{1,2}$ are positive parameters, and the smooth function $f(x)$ satisfies the condition

$$
(f(x)+k x) x<0, \quad \text { for }|x|>x_{\mathrm{sec}} .
$$


We study first the stability properties of the single Lur'e system written in the form of Eq. (17).

Lemma 4.1. The system (17) is dissipative, i.e. there exists a ball $\|(x, y, z)\|<B$ containing an attractor of the system (17) such that the domain $\|(x, y, z)\|>B$ belongs to an exterior part of the basin of attraction of $B$.

Proof. The directing Lyapunov function

$$
V=-\int_{x_{0}}^{x} \frac{f(\xi) d \xi+\left(y^{2}+z^{2}\right)}{2}
$$

has the derivative along the solution curves of Eq. (17)

$$
\dot{V}=-\left(f^{2}+\lambda y^{2}+\lambda z^{2}\right)+\text { wedge terms }
$$

which is negative definite for some region $f^{2}+\lambda y^{2}+$ $\lambda z^{2}>\tilde{B}$ embedded into the domain $\|x, y, z\|>B$ due to Eq. (18).

Consider now a 3-D lattice of diffusively coupled Lur'e systems with zero-flux BC written in the form similar to Eqs. (2)-(17)

$$
\left\{\begin{array}{l}
\dot{x}=f(x)+y+z+\varepsilon_{1} \triangle_{i}+\varepsilon_{2} \triangle_{j}+\varepsilon_{3} \triangle_{k} \\
\dot{y}=b_{1} f(x)-\lambda y-\alpha z \\
\dot{z}=b_{2} f(x)+\omega y-\lambda z
\end{array}\right.
$$

where $x=x_{i, j, k}, y=y_{i, j, k}$, and $z=z_{i, j, k}, i=\overline{1, N}_{1}$, $j=\overline{1, N}_{2}, k=\overline{1, N}_{3}$.

The system (20) has an absorbing domain which is the topological product of the balls $\|(x, y, z)\|<B$ along all the states of the lattice. This statement can be obtained with the help of a directing Lyapunov function that is a sum of the functions (19) written for each oscillator of the 3-D lattice.

Similar to the system (6), we consider the auxiliary system

$$
\left\{\begin{array}{l}
\dot{x}=-a(t) x+y+z \\
\dot{y}=\tilde{b}_{1}(t) x-\lambda y-\alpha z \\
\dot{z}=\tilde{b}_{2}(t) x+\omega y-\lambda z
\end{array}\right.
$$

Here, new coordinates $x, y, z$ play the role of the coordinates $\Phi$ and $\Psi$ of Eq. $(5) . \tilde{b}_{1,2}(t)=b_{1,2} f^{\prime}(\tilde{x}(t))$ and $\tilde{x}(t)$ are defined similar to the vector $B(t)$ and the scalar $x^{*}(t)$ of the general variation equations (5), respectively. $a(t)>0$ is a coefficient standing instead of $f^{\prime}(\tilde{x}(t))$.
Lemma 4.2. Suppose that

$$
\begin{aligned}
& a(t)>a_{0} \\
& \quad \equiv \lambda^{-1}\left(1+\max \left\{\tilde{b}_{1}(t), \tilde{b}_{2}(t)\right\}\right)^{2} \text { and } \lambda>|\omega-\alpha| .
\end{aligned}
$$

Then the auxiliary system (21) is globally asymptotically stable.

Proof. The Lyapunov function

$$
V=x^{2}+y^{2}+z^{2}
$$

has a negative definite derivative with respect to the trajectories of the system (21) under the condition (22). Hence the system (21) is globally asymptotically stable.

Proposition 4.1. As it follows from the general statements of Theorem 3.1 and Lemma 4.2, the sufficient conditions of synchronization of oscillators in each of three directions of the lattice (20) attain the form

$$
\begin{aligned}
\varepsilon_{m} & >\frac{\left(a_{0}+b\right)}{4 \sin ^{2}\left(\frac{\pi}{2 N_{m}}\right)}, \quad m=1,2,3, \\
a_{0} & =\lambda^{-1}\left(1+\max _{|x|<x_{\mathrm{sec}}}\left\{\tilde{b}_{1}(t), \tilde{b}_{2}(t)\right\}\right)^{2}, \\
b & =\max _{|x|<x_{\mathrm{sec}}} f_{x}^{\prime}(x)
\end{aligned}
$$

where $x_{\mathrm{sec}}$ is defined in Eq. (18).

We shall provide now numerical simulations in order to confirm the general results for a concrete form of the Lur'e system. For the numerical study we consider the lattice (1) of $x$-coupled Lur'e systems, where the individual dynamics of each oscillator of the lattice is described by the dynamical system

$$
\left\{\begin{array}{l}
\dot{x}=-\alpha f(x)+y-\alpha z \\
\dot{y}=b_{1} f(x)+a_{11} y+a_{12} z \\
\dot{z}=b_{2} f(x)+a_{21} y+a_{22} z
\end{array}\right.
$$

where $\alpha=10.5, b_{1}=-110.17, b_{2}=10.5, a_{11}=$ $-3.8, a_{12}=-35.25, a_{21}=0.36, a_{22}=2.78$ are fixed parameters and the nonlinear smooth function $f(x)=5\left(x^{3}-9 x / 4\right) / 100$. This form of the function $f(x)$ and the corresponding parameter values are chosen to lead the system (24) to a form of the generalized Chua circuit [Madan, 1993] and to provide the existence of a double-scroll attractor defining the chaotic behavior of the individual oscillator of the lattice. 


\subsection{2-D lattice}

We study numerically the spatiotemporal dynamics of the square lattice (3) with $\varepsilon_{1}=\varepsilon_{2}=\varepsilon$ and the order of appearance of possible cluster synchronization modes when increasing the coupling parameter $\varepsilon$ from zero.

\subsubsection{Zero-flux BC}

\section{$6 \times 6$ lattice}

Figure 2 shows snapshots of the established cluster synchronization regimes for different coupling strengths. Different colors represent different ranges of amplitudes of $x_{i, j}(t)$. With increasing coupling, the cluster synchronization $M^{\mathrm{cl} / \mathrm{des}} \equiv M^{r}(3,6)$ first becomes stable $(\varepsilon=0.47)$ and the corresponding cluster synchronization regime is observed in the lattice [see Fig. 2(a, left)]. The oscillators synchronize in pairs around the middle of the vertical columns and desynchronize in the horizontal rows of the lattice. For $\varepsilon=0.68$, the invariant manifolds $M^{+}(21)$ and $M^{-}(21)$ become stable and define cluster synchronizations with respect to the principal and secondary diagonals, respectively. Realization of a particular of these two cluster modes depends on the initial conditions. For initial conditions taken from the basin of attraction of $M^{-}(21)$, the corresponding spatiotemporal regime arises [see Fig. 2(a, right)] and remains stable in some region of coupling parameter.

The individual Lur'e system has odd symmetry. Therefore the lattice system (3) with a Lur'e system as the individual oscillator is invariant under the involution $\left(x_{i, j}, y_{i, j}, z_{i, j}\right) \rightarrow\left(-x_{i, j},-y_{i, j},-z_{i, j}\right)$. This fact allows the lattice system (3) to have antiphase cluster synchronized oscillations, defined, according to Proposition 2.6, by the antiphase synchronization manifolds $M_{t}^{\times}\left(n_{1}, n_{2}\right)$. For $\varepsilon=0.89$, the cluster manifold $M_{t}^{r}(1,3)$ is indeed stable and defines a spatiotemporal regime under which the oscillators of the lattice are completely inphase synchronized in the vertical columns and the columns are antiphase synchronized in pairs. That is, the first vertical column is antiphase synchronized with the last one, and so forth [see Fig. 2(b)]. With further increase in coupling $\varepsilon=1.2$, the cluster inphase synchronization manifold $M^{\text {syn/des }} \equiv M^{r}(1,6)$ becomes stable and defines full synchronization of the rows and desynchronization of the columns [see Fig. 2(c, left)]. Figure 2(c, right) shows a multiperiodic attractor defining the temporal behavior of the $(3,3)$ oscillator in the regime of the sixcluster synchronization. Obviously, the inphase synchronization manifold $M^{\text {des/syn }} \equiv M^{c}(6,1)$ is also stable and corresponds to full synchronization of the columns and desynchronization of the rows. With final increase of the coupling, the manifold $M(1)$ becomes stable and synchronization of all oscillators of the lattice takes place.

\section{$7 \times 7$ lattice}

We present the results of our computer simulations of cluster synchronization for the square lattice of coupled Lur'e systems with $N=7$ to show that the realization of cluster synchronization modes in this case differs from that of the previous case $N=6$.

For $\varepsilon=0.83$, the invariant manifold $M^{ \pm}(16)$ becomes stable and defines cluster synchronization of the oscillators with respect to the principal and secondary diagonals of the square lattice [see Fig. 3(a)]. With further increase in coupling $(\varepsilon=0.96)$, this cluster pattern decays and the invariant manifold $M^{\text {des/syn }} \equiv M^{c}(7,1)$ becomes stable [see Fig. 3(b)] and defines synchronization of the columns of the lattice and desynchronization of the rows. Under a further increase of the coupling $(\varepsilon=1.01)$, this spatiotemporal synchronization regime gradually develops into the cluster defined by the next manifold from the embedding (4), namely $M^{\mathrm{cl} / \mathrm{syn}} \equiv M^{c}(4,1)$. Here, the oscillators are completely synchronized in the rows and the rows are synchronized in pairs around the middle row of the lattice [see Fig. 3(c)]. Finally, for a sufficiently large $\varepsilon$, full synchronization occurs in the system.

It appeared that not all cluster synchronization patterns, defined by the manifolds from the embedding (4), are stable in this concrete coupled system with zero-flux BC. We shall now consider the 2-D lattice system of coupled Lur'e systems with periodic $\mathrm{BC}$. In this case each oscillator of the $2-\mathrm{D}$ lattice may be considered as the first one so that all Propositions for the existence of linear invariant manifolds may be reformulated with respect to this element and the additional invariant manifolds exist. Therefore we expect to observe the additional stable cluster synchronization modes.

\subsubsection{Periodic $B C$}

\section{$7 \times 7$ lattice}

With increasing coupling between the oscillators, $\varepsilon=0.51$, the cluster synchronization manifold 

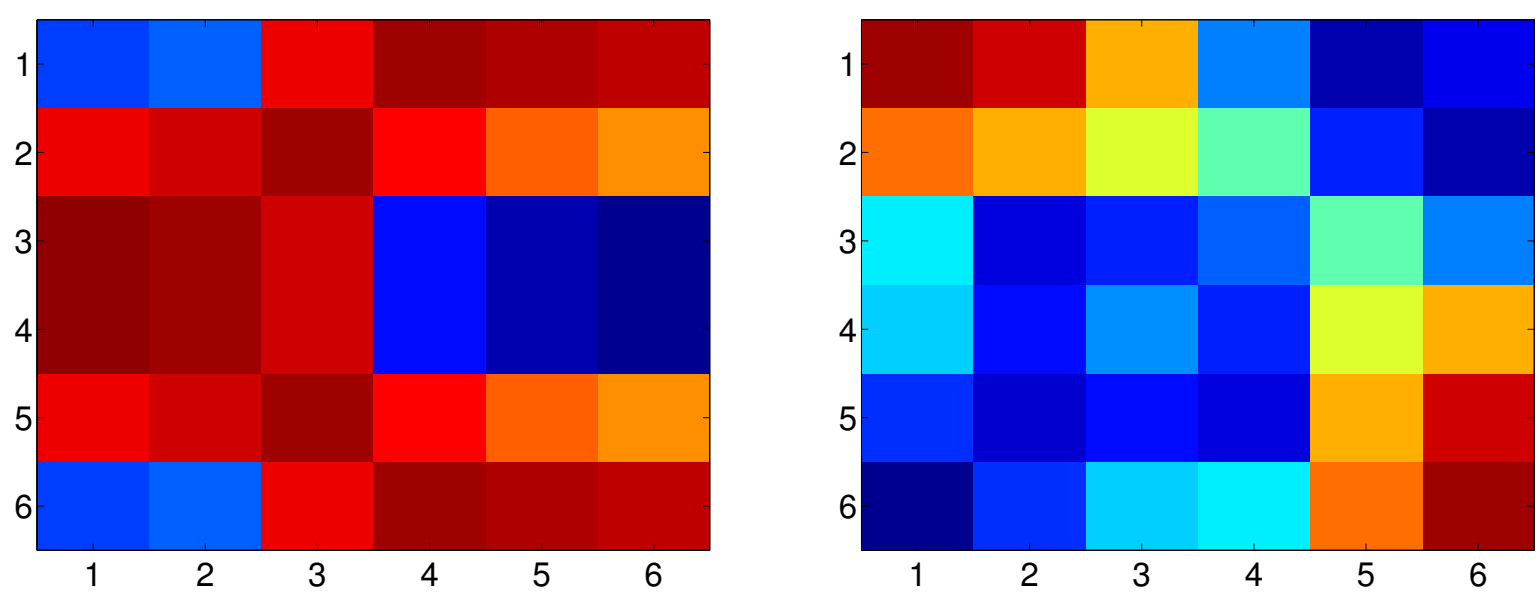

(a)
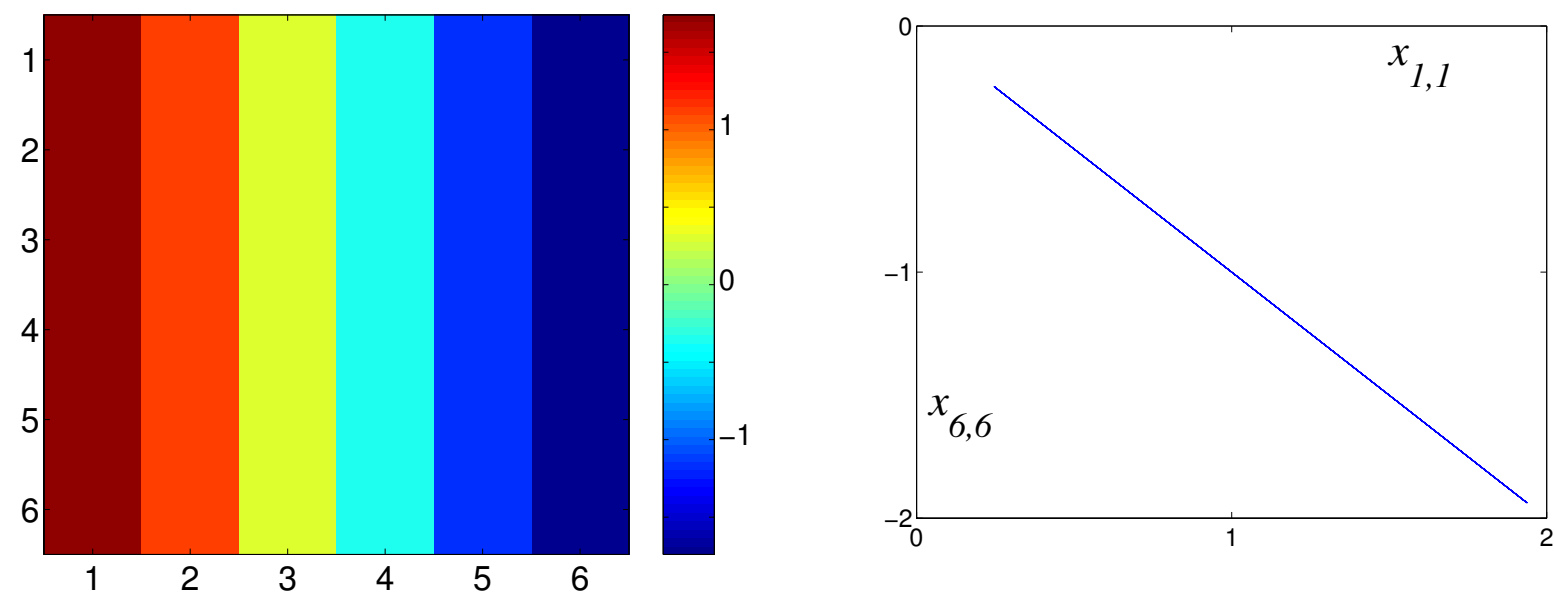

(b)
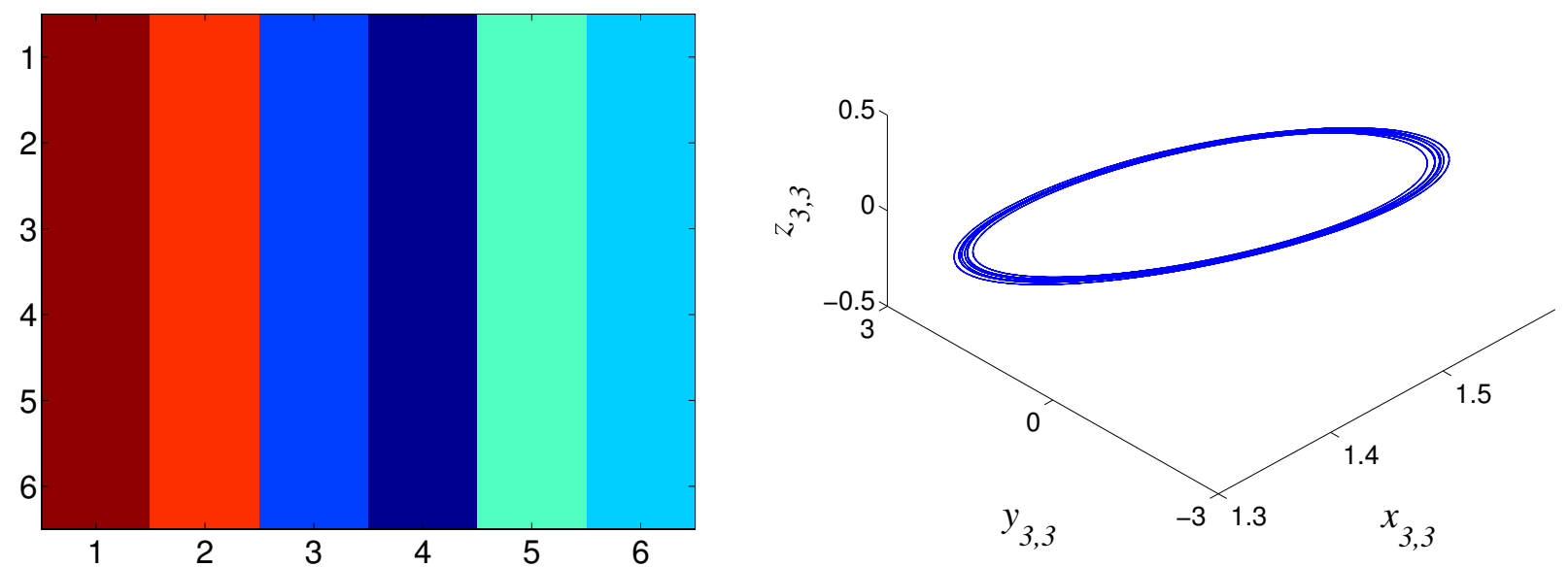

(c)

Fig. 2. Lattice of coupled Lur'e systems $(6 \times 6)$ (zero-flux BC). (a) Rows of nonsynchronized oscillators are synchronized in pairs $(\varepsilon=0.47)$ (left). Synchronization with respect to the secondary diagonal $(\varepsilon=0.68)$ (right). (b) Columns of synchronized oscillators are antiphase synchronized in pairs $(\varepsilon=0.89)$. The bar displays the range of changing of $x_{i, j}$-amplitudes from blue to red. The opposite colors with respect to " 0 " in the bar define antiphase synchronized $x_{i, j}$-amplitudes of oscillators (left). Antiphase synchronization between the $(1,1)$ and $(6,6)$ oscillators (right). (c) Columns of synchronized oscillators are desynchronized $(\varepsilon=1.2)$ (left). Attractor defining the dynamics of the $(3,3)$ oscillator in the regime of the six-cluster synchronization (right). 


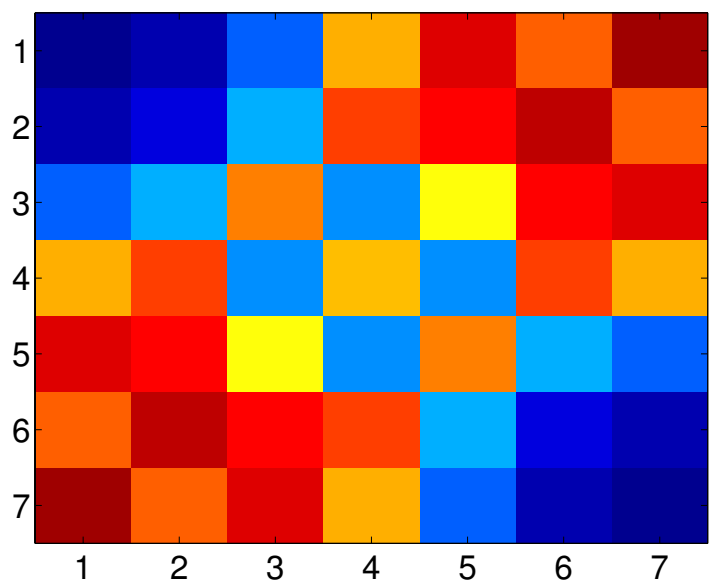

(a)

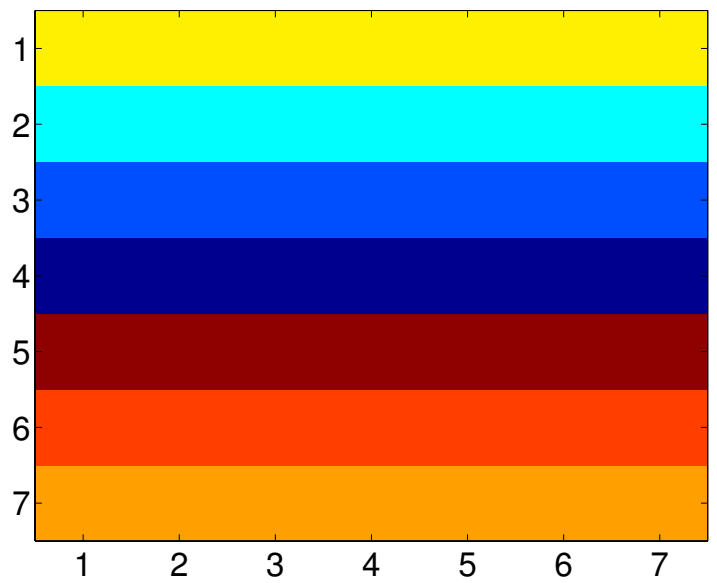

(b)

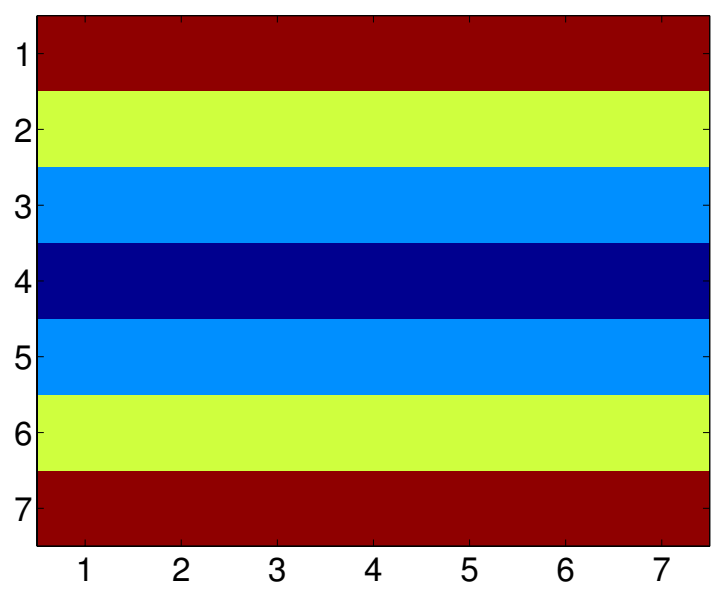

(c)

Fig. 3. Lattice of coupled Lur'e systems $(7 \times 7)$ (zero-flux BC). Snapshots of established cluster synchronization modes for different coupling strengths. (a) Oscillators synchronize with respect to the principal and secondary diagonals of the lattice $(\varepsilon=0.83)$. (b) Oscillators synchronize in rows $(\varepsilon=0.96)$. (c) Rows are synchronized in pairs around the fourth unsynchronized row $(\varepsilon=1.01)$.
$M_{s}^{*}(10)$ becomes stable [see Fig. 4(a, left)]. Here, the nonsynchronized oscillator with the index $(2,5)$ plays the role of the middle element of the 2-D lattice with zero-flux BC. Oscillators synchronize in groups simultaneously with respect to the second row, the fifth column, and with respect to two diagonals passing through the $(2,5)$ element. The two diagonals are obviously continued due to periodic $\mathrm{BC}$. For $\varepsilon=0.54$, a cluster synchronization mode defined by the invariant manifold $M_{s}^{r}(4,7)$ becomes stable and shows cluster synchronization of the horizontal rows of the lattice around the fourth row and a desynchronization pattern between the vertical columns [Fig. 4(a, right)]. For $\varepsilon=0.56$, another cluster synchronization mode arises, where oscillators of the lattice are synchronized in pairs with respect to the diagonal passing through the elements with the indexes $(1,6)$ and $(6,1)$ [see Fig. 4(b)]. The existence of this mode is defined by the invariant manifold $M^{+}(d)$, where $d=N(N+1) / 2=28$ is the number of independent clusters. With further increased coupling $\varepsilon=0.57$, the cluster synchronization manifold $M_{s}^{r}(1,4)$ becomes stable, whereas the full synchronization manifold $M(1)$, embedded into $M_{s}^{r}(1,4)$, remains unstable and the corresponding cluster synchronization occurs [see Fig. 4(c, left)]. The oscillators synchronize within the vertical columns, and the columns are synchronized in pairs around the third column (periodic BC make this situation possible). Figure 4(c, right) presents a chaotic attractor defining the temporal behavior of the $(1,7)$ oscillator in the regime of the four-cluster synchronization. We note that the individual Lur'e system with the chosen individual parameters exhibits a double-scroll attractor in the absence of coupling but does the only "one-scroll" attractor in the regime of the cluster synchronization when the coupling is introduced.

Finally, for a sufficiently large $\varepsilon$, full synchronization of all oscillators of the lattice arises.

We note that the regions of stability of the obtained cluster synchronization regimes often intersect and define the multistability of cluster synchronization. Thus one can find the different stable modes for different initial conditions for the same value of the coupling parameter $\varepsilon$.

\subsection{3-D lattice}

Let us study the onset of full synchronization through the sequence of appearance of cluster 

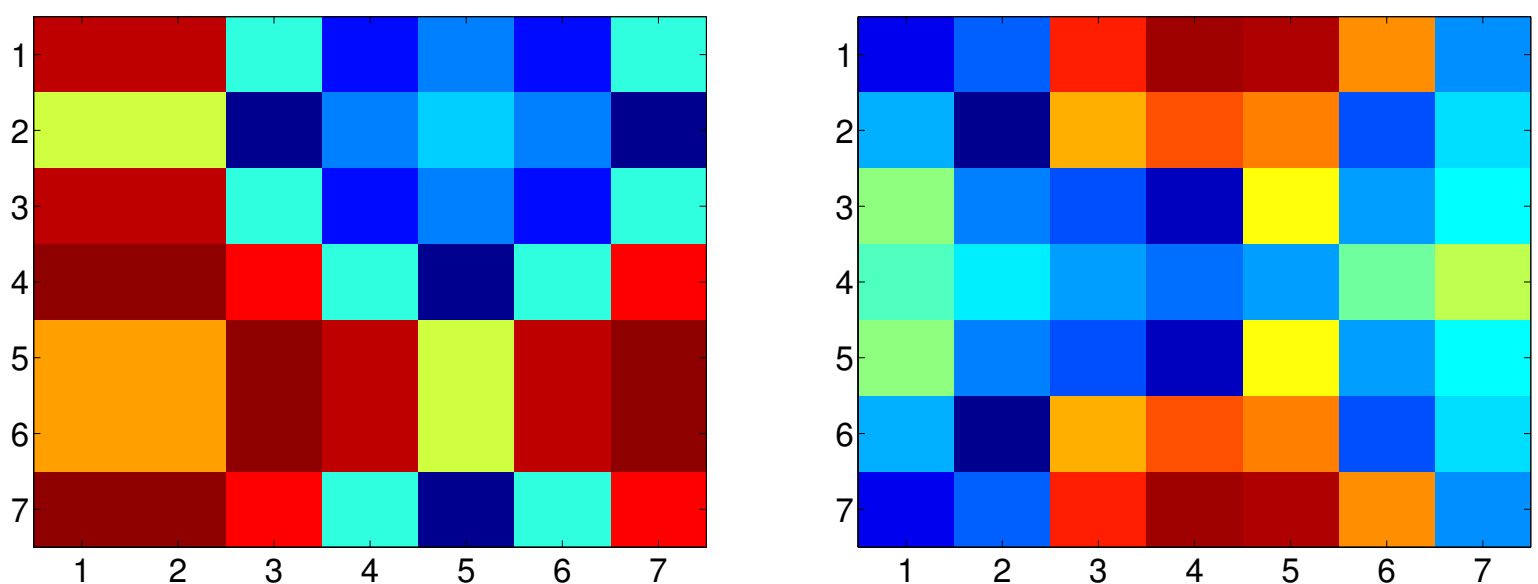

(a)

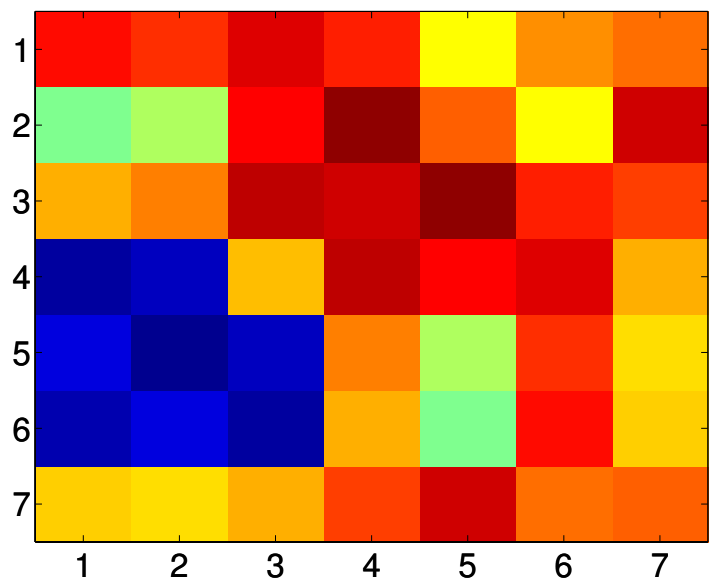

(b)
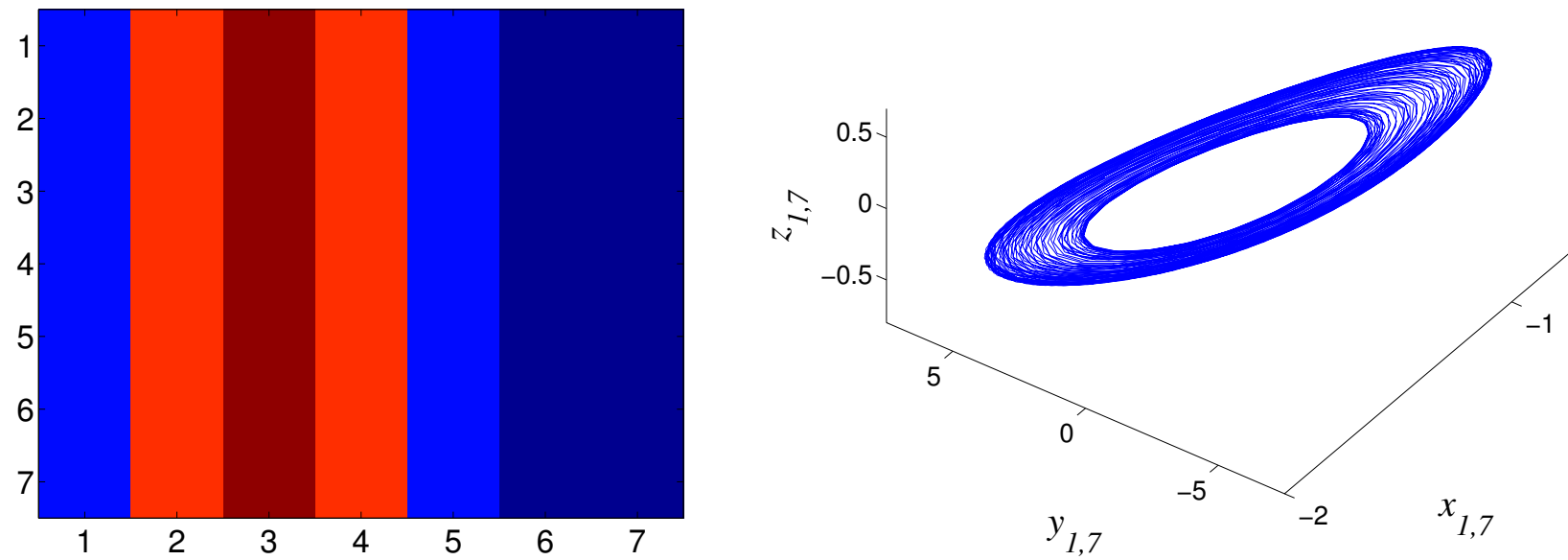

(c)

Fig. 4. Lattice of coupled Lur'e systems $(7 \times 7)$ (periodic BC). (a) Oscillators synchronize with respect to the second row, the fifth column, and with respect to two diagonals passing through the $(2,5)$ element $(\varepsilon=0.51)$ (left). Rows synchronize in pairs around the fourth row $(\varepsilon=0.54)$ (right). (b) Synchronization with respect to the diagonal passing through the $(1,6)$ and $(6,1)$ elements $(\varepsilon=0.56)$. (c) Columns are synchronized in pairs around the third column $\varepsilon=0.57$ (left). Chaotic attractor defining the temporal behavior of the $(1,7)$ oscillator in the regime of four-cluster synchronization (right). 

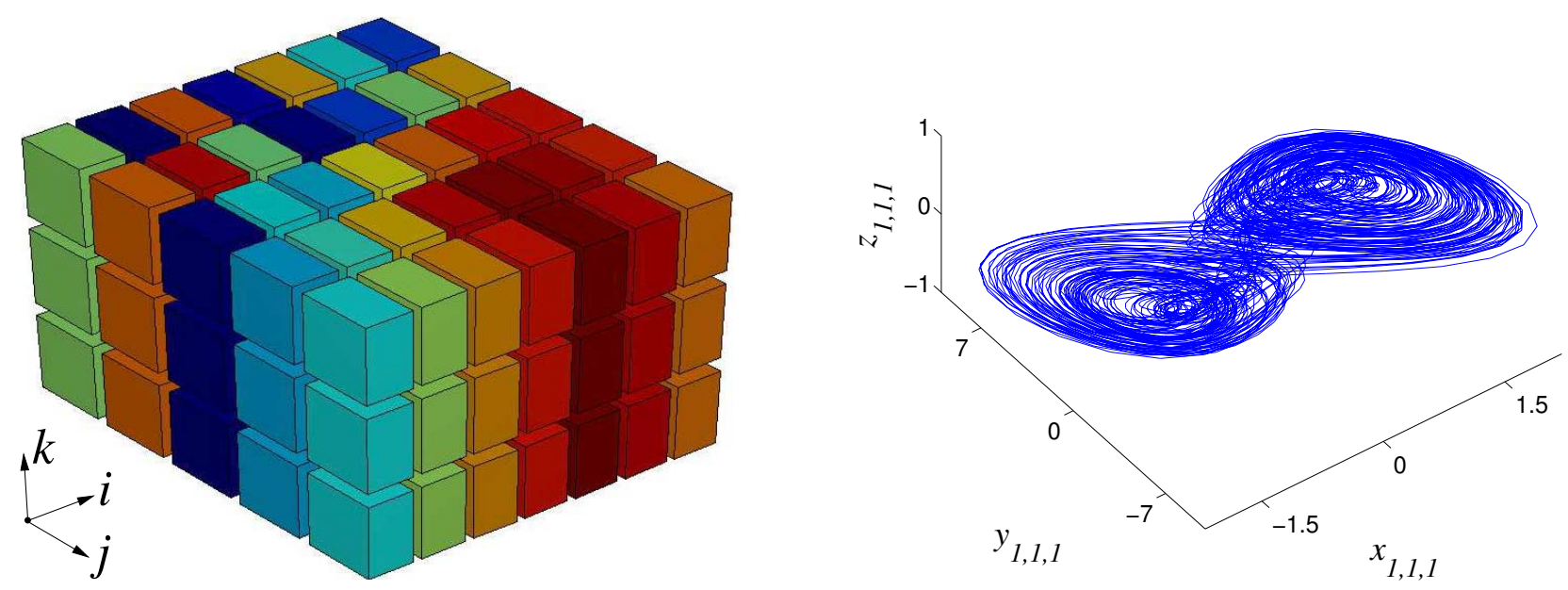

(a)

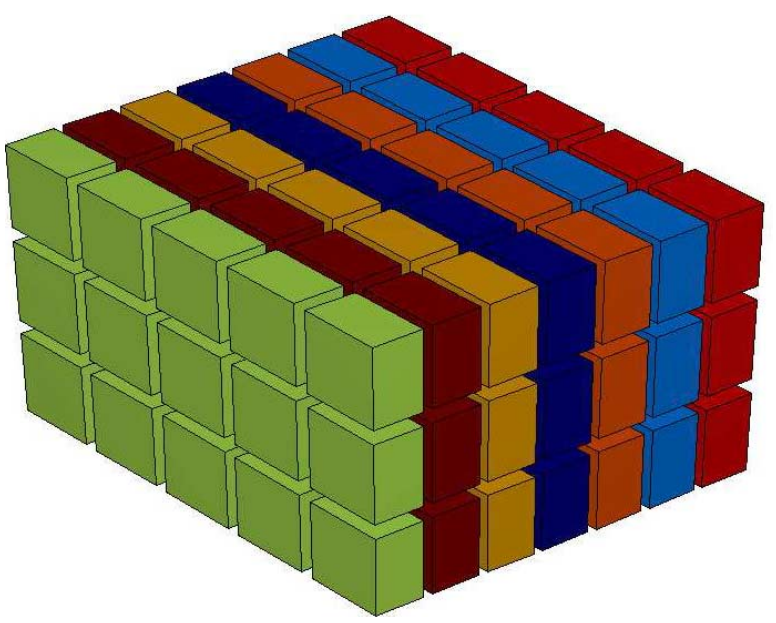

(b)

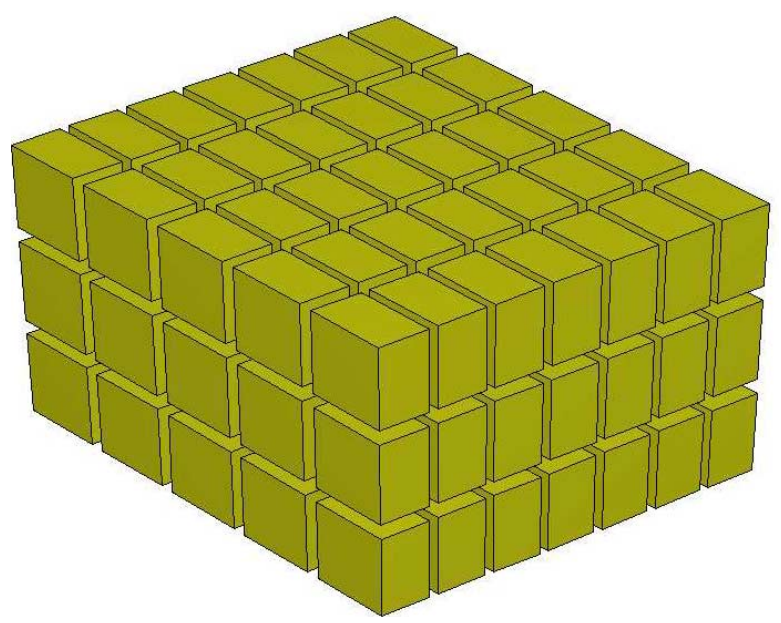

(c)

Fig. 5. 3-D lattice of coupled Lur'e systems $(7 \times 5 \times 3)$. (a) Chaotic synchronization of 2 -D lattices in the $k$ th direction $(\varepsilon=1.15)$ (left). Double-scroll attractor defining the chaotic temporal dynamics of the $(1,1,1)$ oscillator in the regime of the 2-D lattice synchronization (right). (b) Sequential synchronization of the columns within the 2-D lattices $(\varepsilon=1.7)$. (c) Full synchronization $(\varepsilon=3.5)$.

synchronization regimes with increasing coupling in the $7 \times 5 \times 3$ lattice of coupled Lur'e systems (20)(24) with zero-flux BC. To reach synchronization of all oscillators of the 3 -D lattice, we shall change the coupling parameters $\varepsilon_{1}, \varepsilon_{2}$, and $\varepsilon_{3}$ along the diagonal $\varepsilon_{1}=\varepsilon_{2}=\varepsilon_{3}=\varepsilon$ in the coupling parameter space.

Figure 5 presents snapshots of established simple cluster synchronization modes for different coupling strengths. Oscillators with identical color shading belong to the same cluster. However, one can meet a few cases where different shadings look similar due to the limitation of the color presentation. In accordance with the conditions (23), the invariant manifold $M^{v}\left(N_{1}, N_{2}, 1\right)$ becomes globally stable with increasing the coupling $(\varepsilon=1.15)$, and chaotic synchronization of the 2-D lattices in the $k$ th direction arises (the direction of the smallest $\left.N\left(N_{3}=3\right)\right)$ [see Fig. 5(a)]. With increased coupling $\varepsilon=1.7$, the invariant manifold $M^{c v}\left(N_{1}, 1,1\right)$ acquires global stability, and the previous cluster synchronization pattern gradually develops into a synchronization mode defining synchronization of the 2-D lattices in the $k$ th direction and synchronization of $2-\mathrm{D}$ lattices in the $j$ th direction (the direction of $N_{2}=5$ ) [see Fig. 5(b)]. Obviously, $M^{c v}\left(N_{1}, 1,1\right) \subset M^{v}\left(N_{1}, N_{2}, 1\right)$. With further increased coupling, the synchronization manifold $M(1)$ becomes stable and synchronization of all oscillators of the 3-D lattice arises [see Fig. 5(c)]. 
This sequence of the cluster synchronization mode appearance may be also interpreted as full synchronization of the $K^{k}$ planes, sequential synchronization of the columns $C_{j}$ within the $K^{k}$ planes, and finally synchronization of the oscillators within the $C_{j}$ columns.

\section{Coupled Rössler Oscillators}

\subsection{2-D lattice}

Let us consider, as the second example of 2-D lattices of coupled dynamical systems with individual chaotic behavior, the square 2-D lattice (3) of $x$-coupled Rössler oscillators with $\varepsilon_{1}=\varepsilon_{2}=\varepsilon$ and zero-flux BC. The individual dynamics of each oscillator of the lattice is described by the dynamical system

$$
\left\{\begin{array}{l}
\dot{x}=-(y+z) \\
\dot{y}=x+a y \\
\dot{z}=b+(x-c) z .
\end{array}\right.
$$

The parameters are $a=0.2, b=0.2, c=5.7$.

In this case the global stability analysis of the synchronization manifolds cannot be used and the synchronization patterns observed for increasing coupling are different.

\subsection{1. $7 \times 7$ lattice}

Figure 6 presents snapshots of the established stable cluster synchronization regimes existing in the coupled system for different coupling strengths and their order of stabilization. With increasing coupling, the full synchronization manifold $M(1)$ first becomes locally stable and attracts the trajectories from its small neighborhood (global stability is impossible due to a singularity of the individual Rössler system and the use of $x$-coupling [Belykh et al., 2000]), and a spatially homogeneous cluster of chaotic oscillators is observed in the system. Thus the cluster synchronization appearance, that may be defined by the embedding (4) for a prime number $N$, has the reverse order.

In fact, with further increased coupling, the full synchronization manifold $M(1)$ loses its local stability but the next embedded manifold of a greater dimension from the embedding (4), the manifold $M^{\mathrm{cl} / \mathrm{syn}} \equiv M^{c}(4,1)$, remains stable $(\varepsilon=0.25)$, and the lattice exhibits full synchronization of the vertical columns and four-cluster synchronization of the horizontal rows [Fig. 6(a, left)]. With increasing coupling $(\varepsilon=0.4)$, the manifold $M^{\mathrm{cl} / \mathrm{syn}}$ in turn loses its stability and there exists a stable cluster regime under which the oscillators remain synchronized within the horizontal rows of the lattice but now there is no cluster synchronization between the rows [Fig. 6(a, right)]. Realization of this cluster regime is defined by the stability of the invariant manifold $M^{\text {des/syn }}=M^{c}(7,1)$ and the simultaneous instability of all other invariant manifolds of less dimensions embedded into it, namely, the invariant manifolds $M(1)$ and $M^{\mathrm{cl} / \mathrm{syn}}$. For $\varepsilon=0.55$, the manifold $M^{\text {des/syn }}$ loses its stability and a cluster synchronization mode, defined by the next manifold $M^{\text {des/cl }} \equiv M^{c}(7,4)$ of a greater dimension from the embedding (4), arises [Fig. 6(b)]. For $\varepsilon=0.58$, the invariant manifold $M_{s}^{*}(10)$ becomes stable. Figure 6(c, left) presents the established cluster regime defined by the manifold $M^{*}(10)$. This spatiotemporal pattern, with chaotic time dependent amplitudes of the individual oscillators, defines ten clusters and synchronization in nine groups of elements. Figure 6(c, right) shows a chaotic attractor defining the temporal behavior of the $(2,2)$ oscillator in the regime of the cluster synchronization.

Finally, with gradual increasing coupling this cluster synchronization regime becomes unstable and develops into a completely unsynchronized pattern defining $7 \times 7$ independent clusters. Thus, the sequence of bifurcations corresponding to the increase of the dimension of cluster synchronization (the number of independent clusters) appears to be broken at this place.

\subsection{2. $31 \times 31$ lattice}

We consider this case of the prime number $N=$ 31 to show that a stable cluster synchronization regime, defined by the invariant manifold $M^{*}((n+$ 1) $(n+2) / 2)$, for $N=2 n+1$ with the number of clusters $d=153$, arises in the system (for $\varepsilon=0.6$ ) and preserves in some wide region of coupling parameter. Figure 7 (top) presents the established cluster synchronization regime defined by the manifold $M^{*}(153)$. The middle element of the lattice, the $(16,16)$ oscillator, remains unsynchronized and defines one separate cluster. Its temporal chaotic behavior is presented in Fig. 7 (bottom).

\subsection{3-D lattice}

We consider the cubic $N \times N \times N$ lattice (2) with $\varepsilon_{1}=\varepsilon_{2}=\varepsilon_{3}=\varepsilon$ and zero-flux $\mathrm{BC}$ and the 

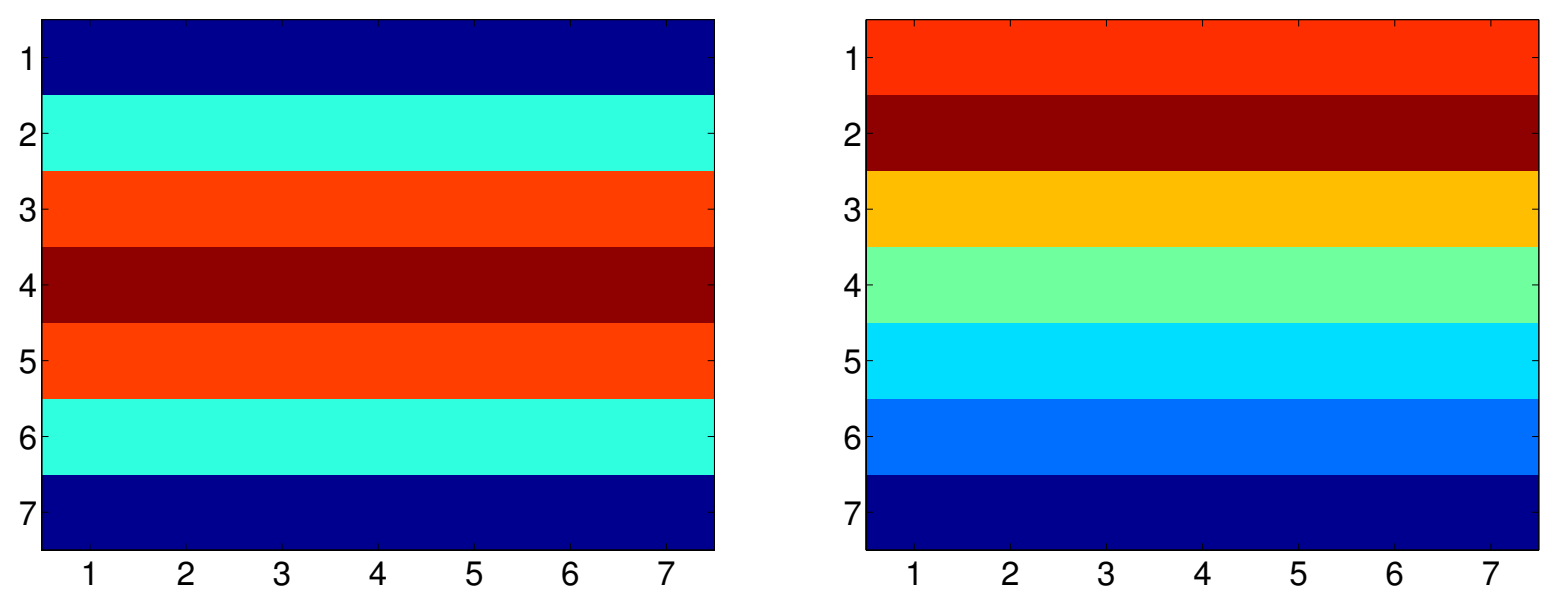

(a)

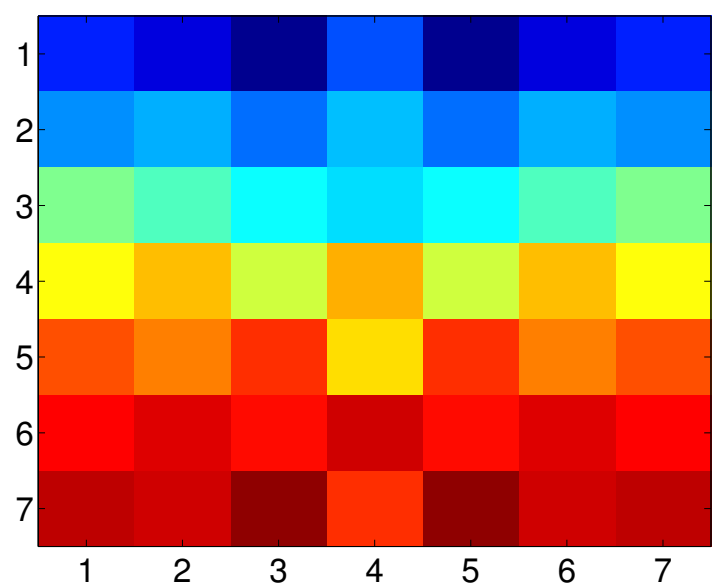

(b)
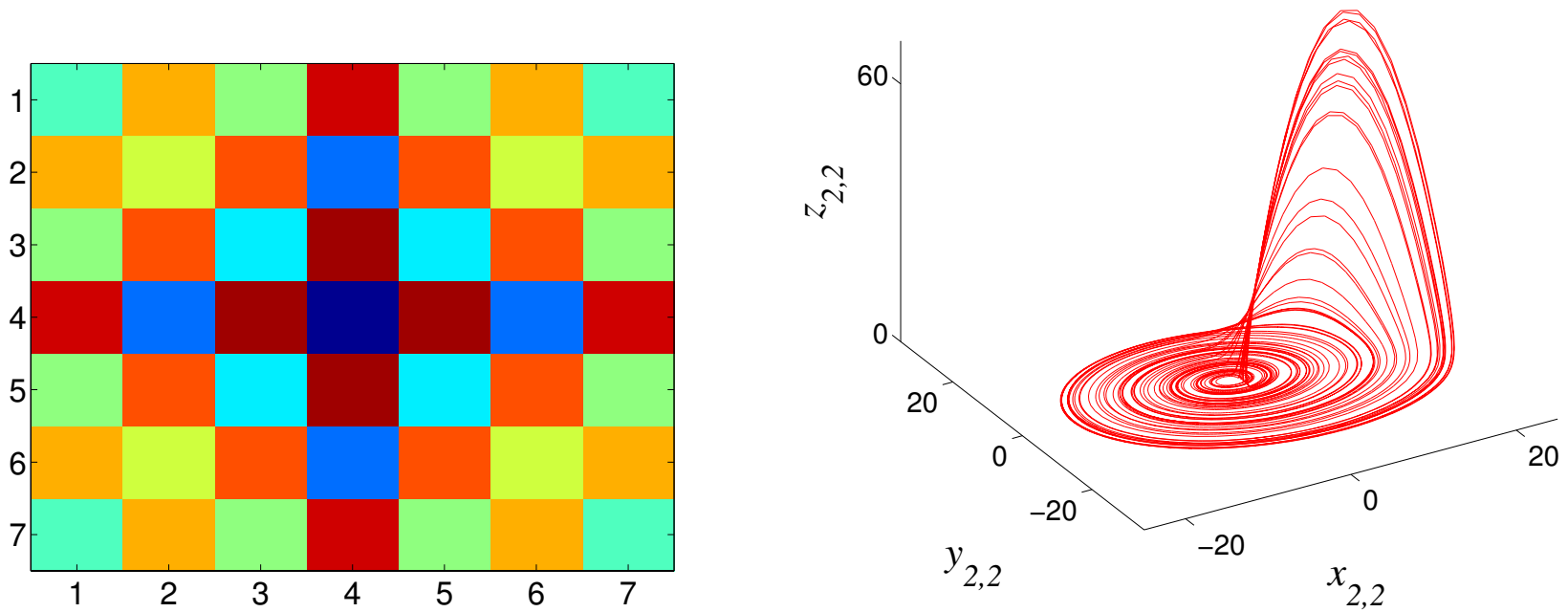

(c)

Fig. 6. 2-D lattice of coupled Rössler systems $(7 \times 7)$. (a) Oscillators synchronize in rows, and the rows synchronize in pairs around the middle row $(\varepsilon=0.25)$ (left). There is no cluster synchronization between the rows of synchronized oscillators $(\varepsilon=0.4)$ (right). (b) The vertical columns synchronize in pairs around the middle column $(\varepsilon=0.55)$. (c) Oscillators synchronize simultaneously with respect to the diagonals and to the middles of the rows and columns of the lattice $(\varepsilon=0.58)$. 

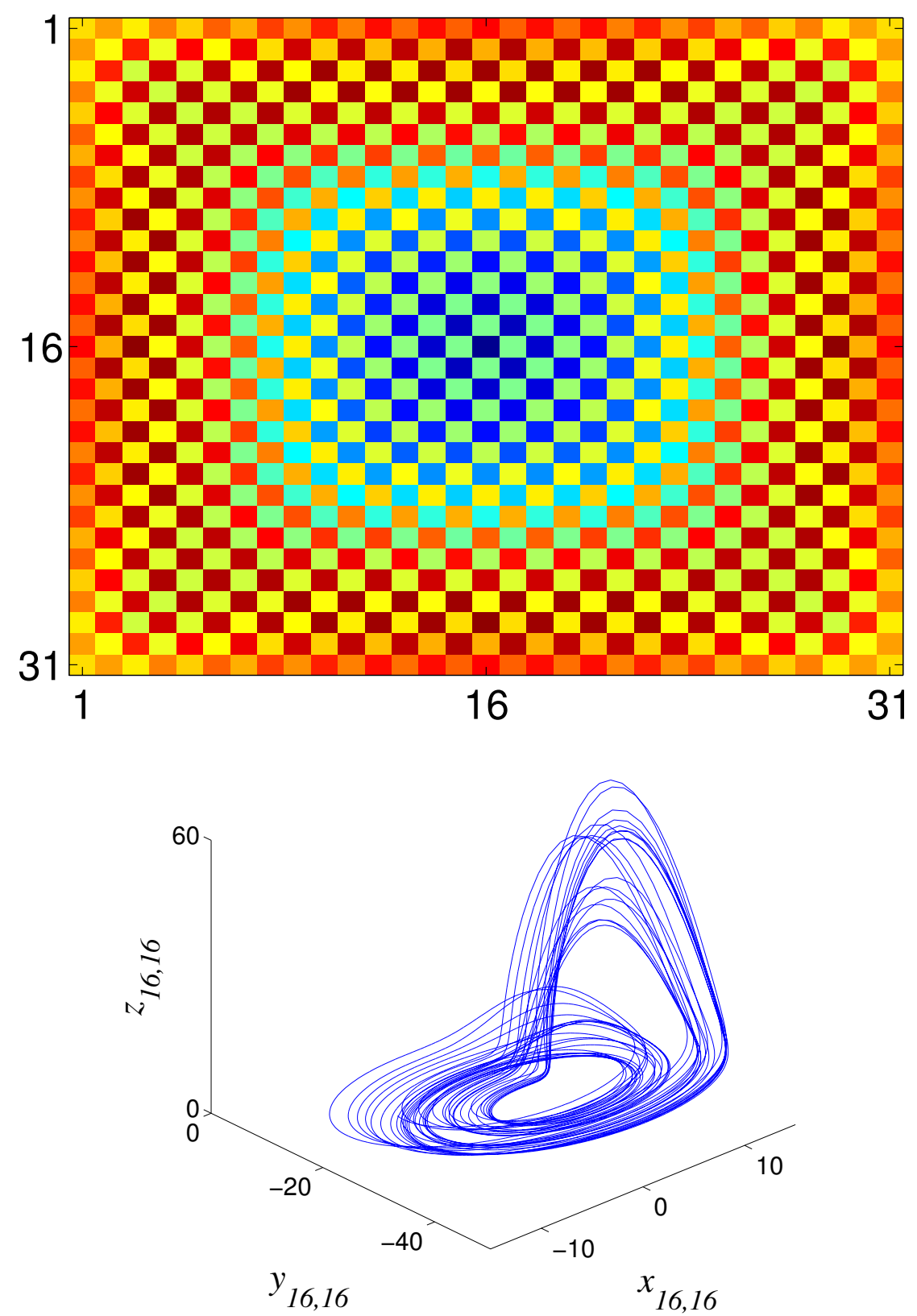

Fig. 7. 2-D lattice of coupled Rössler systems $(31 \times 31)$. Cluster synchronization, similar to that of Fig. $6(\mathrm{c})$, defined by the manifold $M^{*}(153)$ (top). Temporal chaotic behavior of the middle nonsynchronized oscillator with the index (16, 16) (bottom).

individual system $X=F(X), X \in R^{3}$ that is introduced by the Rössler oscillator (25).

\subsection{1. $3 \times 3 \times 3$ lattice}

We omit the results of numerical analysis of the sequence of stabilization of possible cluster synchronization modes with changing coupling between the oscillators and use this case of $N=3$ as an illustrating example of the structure of the coupling (nearest-neighbor interaction) and the existence of the stable four-cluster synchronization, defined by the symmetrical invariant manifold $M_{s}^{*}(4)$. Figure 8 presents this cluster synchronization mode where the oscillators, denoted by balls with the same color, are related to one cluster. The middle $(2,2,2)$ oscillator, being the core of the volume lattice, is nonsynchronized and defines a separate cluster $(\varepsilon=0.5)$.

It is interesting to note here, that the cluster manifold $M_{s}^{*}(d)$ defines synchronization between the oscillators which are not involved in direct coupling interaction. 

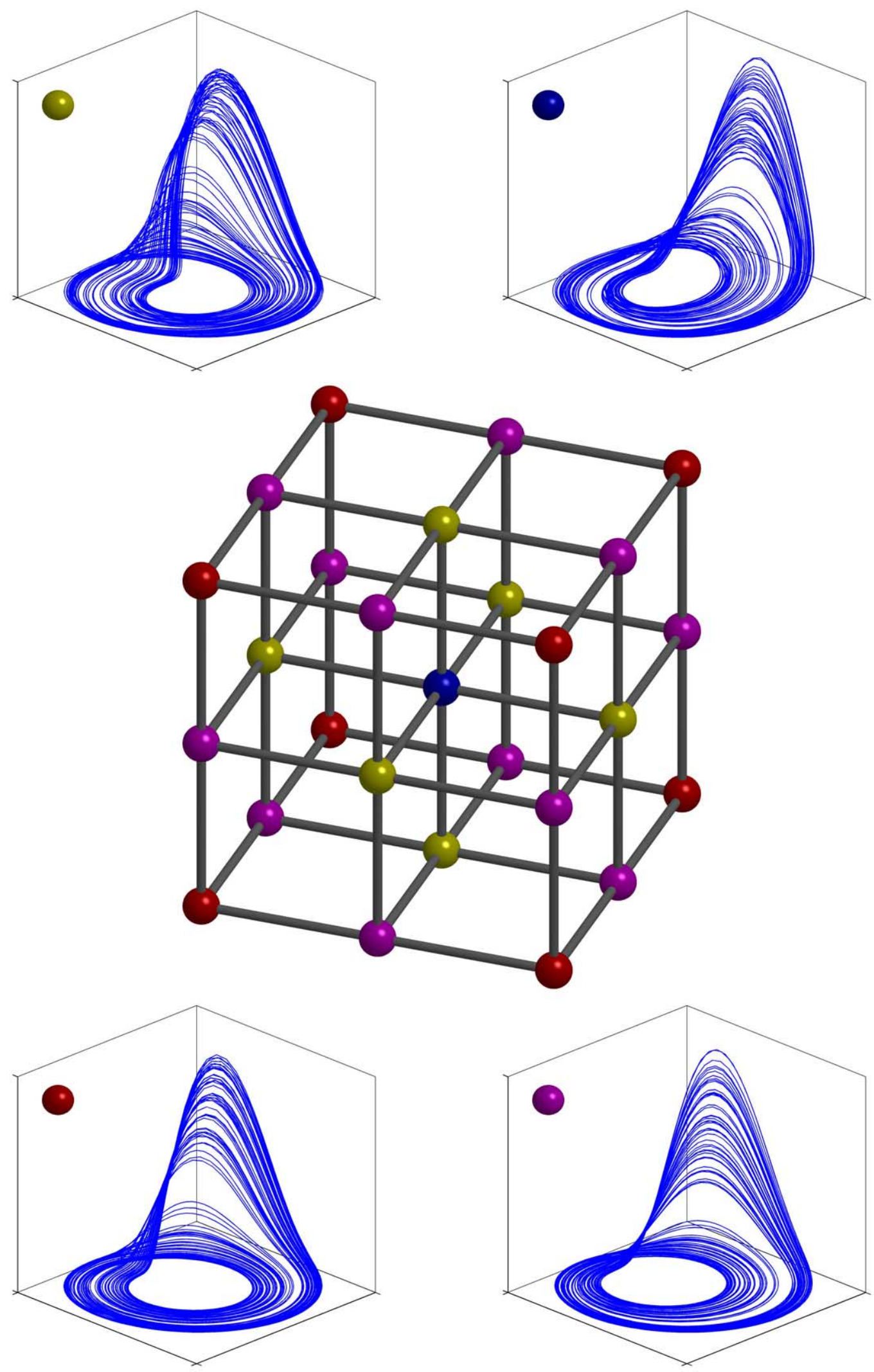

Fig. 8. 3-D lattice of coupled Rössler systems $(3 \times 3 \times 3)$. Oscillators denoted by balls with the same color belong to one cluster. The temporal behavior of each cluster is depicted in the four frames. 


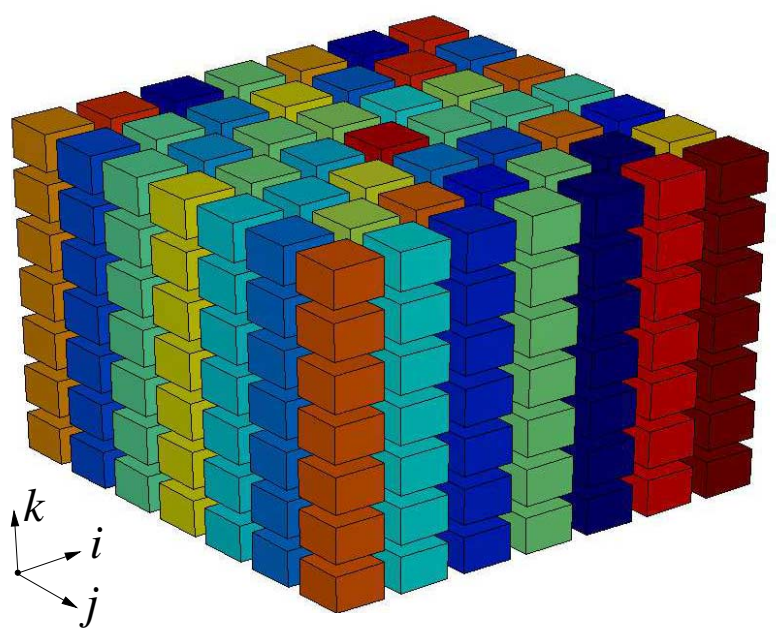

(a)

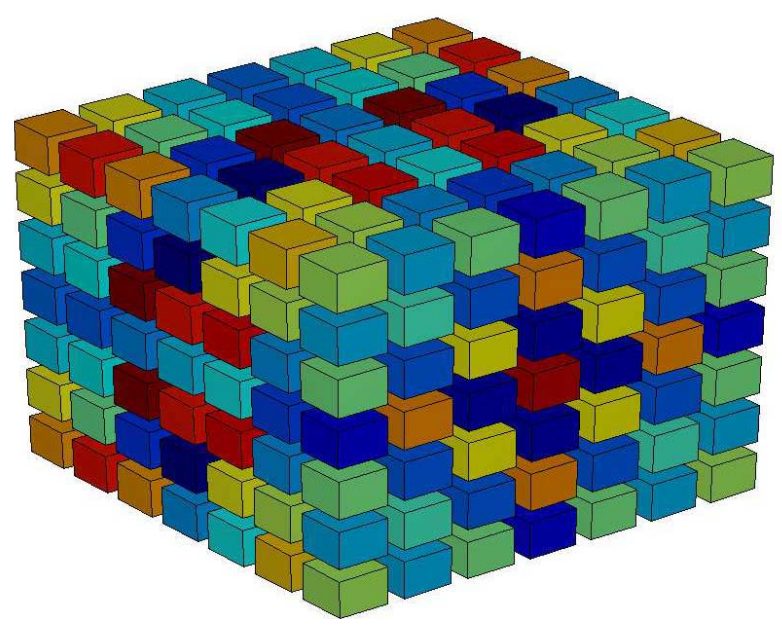

(c)

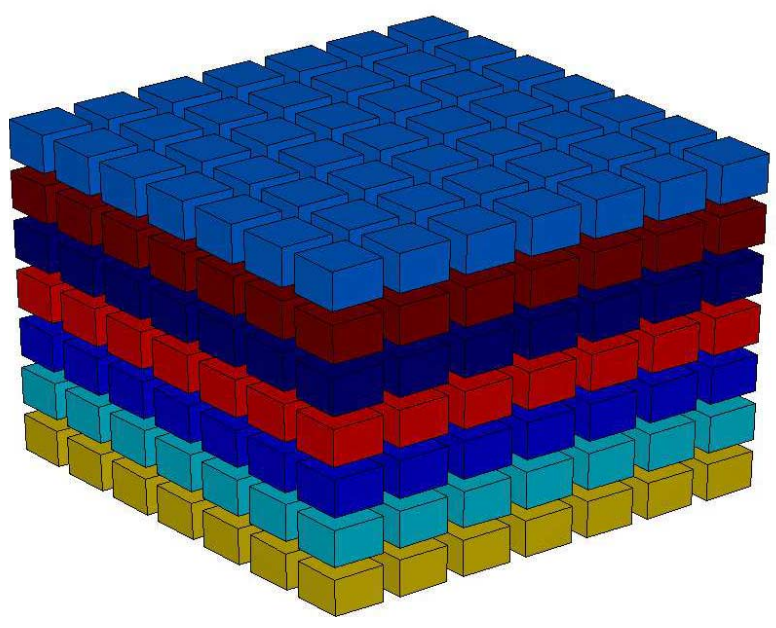

(b)

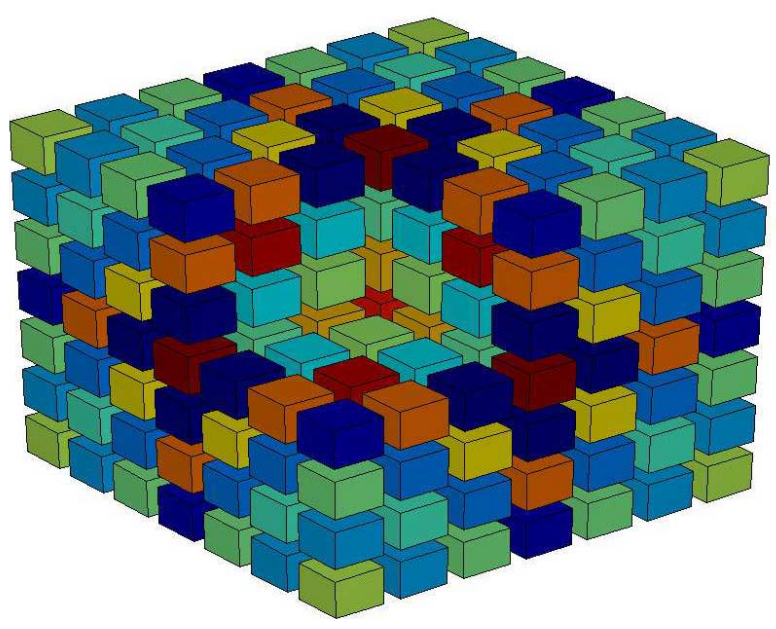

(d)

Fig. 9. 3-D lattice of coupled Rössler systems $(7 \times 7 \times 7)$. (a) 2-D lattices of nonsynchronized oscillators synchronize in one lattice direction $(\varepsilon=0.25)$. (b) Sequential synchronization of the oscillators within the 2-D synchronized lattices $(\varepsilon=0.3)$. (c) Symmetrical cluster synchronization in each plane in one lattice direction and out of synchronization between the planes $(\varepsilon=0.38)$. (d) Oscillators synchronize with respect to all diagonals sections and to the middle elements of the rows, columns and verticals, simultaneously $(\varepsilon=0.4)$. The "cavern" reveals the internal cluster structure and the core nonsynchronized $(4,4,4)$ oscillator depicted by the bright red color.

\subsection{2. $7 \times 7 \times 7$ lattice}

Figure 9 presents a sequence of the appearance of cluster chaos synchronization modes with changing coupling. For $\varepsilon=0.25$, the 2 -D lattices of nonsynchronized oscillators become synchronized in one lattice direction [see Fig. 9(a)] (the manifold $M^{v}(N, N, 1)$ becomes stable). For $\varepsilon=0.3$, sequential synchronization of the rows of the 2-D synchronized lattices arises [see Fig. 9(b)]. For $\varepsilon=$ 0.38 , there arises cluster synchronization where the stable symmetrical ten-cluster pattern is observed in each plane in one lattice direction (similar to that of the 2-D case, see Fig. 6(a) but there is no synchronization between the planes [see Fig. 9(c)]. Therefore the number of clusters of the 3-D lattice equals $d=10 \cdot 7=17$. Obviously similar clusters, realized in other lattice directions, are also stable in the same range of coupling strength. This is due to the cubic lattice symmetry. For $\varepsilon=0.4$, the stable cluster synchronization pattern defined 
by the manifold $M_{s}^{*}(d)$ with the number of clusters $d=(n+1)\left(n^{2}+n+2\right) / 2=28$ for $N=2 n+1=7$, is observed. Oscillators synchronize simultaneously with respect to all diagonal sections and to the middle of the rows, columns and verticals of the 3-D lattice. The core $(4,4,4)$ element remains nonsynchronized. Figure $9(\mathrm{~d})$ presents a snapshot of this spatiotemporal pattern, with chaotic time dependent amplitudes of the individual oscillators. The "cavern" in the cubic lattice allows to see the internal clusters and the core nonsynchronized oscillator depicted by the bright red color. With further increase in coupling, this pattern decays into complete desynchronization.

\section{Conclusions}

We have described the family of cluster synchronization manifolds for a 3-D lattice of diffusively coupled dynamical systems and studied the stability of the manifolds. These embedded manifolds come from the symmetries of coupling, boundary conditions and, in particular, from the cubic (square) lattice symmetry, independent of the dynamics of the individual cells. They serve as a frame for the possible dynamical behaviors of the coupled systems in phase space, defining, in particular, the strict set of cluster synchronized modes that can occur in the lattice.

The main conclusion is that when studying coupled dynamical systems, one should not only concentrate on their equilibrium points, but one should pay equal attention to the cluster synchronization manifolds.

We also conclude the present investigation by mentioning that our results on the existence of the cluster synchronization manifolds in 2-D and 3-D lattices are also valid for the vector diffusive coupling between the oscillators and for 2-D and 3-D lattices of locally coupled maps. We believe that our general results will serve to a better understanding of the phenomenon of cluster synchronization in the lattices composed of a large number of diffusively coupled continuous (or discrete-time) oscillators where numerical analysis had been mainly applied.

Finally, we note that all the invariant synchronization manifolds discovered in the paper, are actually hyperplanes. But we used the more general term "manifold" in order to emphasize the property of persistence of the stable manifolds under small perturbations in which case the perturbed synchro- nization hyperplanes become nonlinear manifolds. These perturbations arise in the case of a parameter mismatch between the oscillators [Afraimovich et al., 1986; Johnson et al., 1998; Belykh et al., 2003].

\section{Acknowledgments}

We are grateful to L. P. Shilnikov, E. Mosekilde, O. de Feo and M. V. Shashkov for helpful discussions and useful comments. I. Belykh and M. Hasler acknowledge the financial support of the Swiss National Science Foundation through Grant No. 2100065268. This work was supported in part by INTAS (Grant No. 01-2061) and RFFI (Grant Nos. 99-0100231 and 02-01-00968).

\section{References}

Afraimovich, V. S., Verichev, N. N. \& Rabinovich, M. I. [1986] "Stochastically synchronized oscillators in dissipative systems," Radiophys. Quant. Electron. 29, 795-803.

Afraimovich, V. S., Chow, S. N. \& Hale, J. K. [1997] "Synchronization in lattices of coupled oscillators," Physica D103, 445-451.

Alexander, J. C., Yorke, J. A. \& You, Z. [1992] "Riddled basins," Int. J. Bifurcation and Chaos 2, 795-813.

Ashwin, P., Buescu, J. \& Stewart, I. [1994a] "Bubbling of attractors and synchronization of chaotic oscillators," Phys. Lett. A193, 126-139.

Ashwin, P., Buescu, J. \& Stewart, I. [1994b] "From attractor to chaotic saddle: A tale of transverse instability," Nonlinearity 9, 703-737.

Belykh, V. N., Verichev, N. N., Kocarev, L. J. \& Chua, L. O. [1993] "On chaotic synchronization in a linear array of Chua's circuits," J. Circuits Syst. Comput. 3(2), 579-589.

Belykh, V. N. \& Mosekilde, E. [1996] "One-dimensional map lattices: Synchronization, bifurcations, and chaotic structures," Phys. Rev. E54, 3196-3203.

Belykh, V. N., Belykh, I. V. \& Hasler, M. [2000] "Hierarchy and stability of partially synchronous oscillations of diffusively coupled dynamical systems," Phys. Rev. E62(5), 6332-6345.

Belykh, V. N., Belykh, I. V. \& Mosekilde, E. [2001] "Cluster synchronization modes in an ensemble of coupled chaotic oscillators," Phys. Rev. E63, 036216.

Belykh, I. V., Belykh, V. N., Nevidin, K. V. \& Hasler, M. [2003] "Persistent clusters in lattices of coupled nonidentical chaotic systems," Chaos 13, 165-178.

Bel'skii, Yu. L. \& Dmitriev, A. S. [1993] "Information transmission using deterministic chaos," Radiotechn. Elektron. 38, 1310-1315.

Chen, G. (ed.) [2000] Controlling Chaos and Bifurcations in Engineering Systems (CRC Press, Boca Raton, FL). 
Chiu, C. H., Lin, W. W. \& Peng, C. C. [2000] "Asymptotic synchronization in lattices of coupled nonidentical Lorenz equations," Int. J. Bifurcation and Chaos 10(12), 2717-2728.

Chow, S. \& Mallet-Paret, J. [1995] "Pattern formation and spatial chaos in lattice dynamical systems Part I," IEEE Trans. Circuits Syst.-I 42, 746-751.

Chua, L.O. \& Yang, L. [1988] "Cellular neural networks: Theory and practice," IEEE Trans. Circuits Syst.-I 35, 1257-1290.

Chua, L. O., Hasler, M., Moschytz, G. S. \& Neirynek, J. [1995] "Autonomous cellular neural networks: A united paradigm for pattern formation and active wave propagation," IEEE Trans. Circuits Syst.-I 42, $559-577$.

Chua, L. O. [1997] "CNN: A vision of complexity," Int. J. Bifurcation and Chaos 7(10), 2219-2425.

Fink, K. S., Johnson, G., Carroll, T., Mar, D. \& Pecora, L. M. [2000] "Three coupled oscillators as a universal probe of synchronization stability in coupled oscillator arrays," Phys. Rev. E61, 5080-5090.

Fradkov, A. L. \& Pogromsky, A. Yu. [1998] Introduction to Control of Oscillations and Chaos (World Scientific, Singapore).

Fujisaka, H. \& Yamada, T. [1983] "Stability theory of synchronized motion in coupled oscillator systems," Progr. Theor. Phys. 69, 32-46.

Hasler, M. [1995] "Engineering chaos for encryption and broadband communication," Phil. Trans. R. Soc. Lond. A353, 115-126.

Hasler, M., Maistrenko, Yu. \& Popovich, O. [1998] "Simple example of partial synchronization of chaotic systems," Phys. Rev. E58(5), 6843-6846.

Heagy, J. F., Carroll, T. L. \& Pecora, L. M. [1994] "Synchronous chaos in coupled oscillator systems," Phys. Rev. E50, 1874-1885.

Johnson, G. A., Mar, T. L., Carroll, T. L. \& Pecora, L. M. [1998] "Synchronization and imposed bifurcations in the presence of large parameter mismatch," Phys. Rev. Lett. 80, 3956-3959.

Josić, K. [2000] "Synchronization of chaotic systems and invariant manifolds," Nonlinearity 13, 1321-1336.

Kaneko, K. [1990] "Clustering, coding, switching, hierarchical ordering, and control in a network of chaotic elements," Physica D41, 137-172.

Kazantsev, V. B., Nekorkin, V. I., Artyuhin, D. V. \& Velarde, M. G. [2001] "Synchronization, re-entry, and failure of spiral waves in a two-layer discrete excitable system," Phys. Rev. E63, 016212.

Lemaître, A. \& Chaté, H. [1999] "Phase ordering and onset of collective behavior in chaotic coupled map lattices," Phys. Rev. Lett. 82, 1140-1143.

Madan, R. N. (ed.) [1993] Chua's Circuit: A Paradigm for Chaos (World Scientific, Singapore).

Mallet-Paret, J. \& Chow, S. [1995] "Pattern formation and spatial chaos in lattice dynamical systems Part II," IEEE Trans. Circuits Syst.-I 42, 752-756.

Nekorkin, V. I., Makarov, V. A., Kazantsev, V. B. \& Velarde, M. G. [1997] "Spatial disorder and pattern formation in lattices of coupled bistable elements," Physica D100, 330-342.

Pecora, L. M. \& Carroll, T. L. [1990] "Synchronization in chaotic systems," Phys. Rev. Lett. 64, 821-824.

Pecora, L. M. [1998] "Synchronization conditions and desynchronization patterns in coupled limit-cycle and chaotic systems," Phys. Rev. E58, 347-360.

Pecora, L. M. \& Carroll, T. L. [1998] "Master stability functions for synchronized coupled systems," Phys. Rev. Lett. 80, 2109-2112.

Pogromsky, A. Yu. \& Nijmeijer, H. [2001] "Cooperative oscillatory behavior of mutually coupled dynamical systems," IEEE Trans. Circuits Syst.-I 48, 152-162.

Rabinovich, M. I., Torres, J. J., Varona, P., Huerta, R. \& Weidman, P. [1999] "Origin of coherent structures in a discrete chaotic medium," Phys. Rev. E60(2), R1130-R1133.

Sherman, A. [1994] "Anti-phase, asymmetric and aperiodic oscillations in excitable cells - I. Coupled bursters," Bull. Math. Biol. 56(5), 811-835.

Thiran, P., Crounse, K. R., Chua, L. O. \& Hasler, M. [1995] "Pattern formation properties of autonomous cellular neural networks," IEEE Trans. Circuits Syst.-I 42, 757-774.

Thiran, P. [1997] Dynamics and Self-Organization of Locally Coupled Neural Networks (Presses Polytechniques et Universitaires Romandes, Lausanne).

Wu, C. W. \& Chua, L. O. [1996] "On a conjecture regarding the synchronization in an array of linearly coupled dynamical systems," IEEE Trans. Circuits Syst.-I 43, 161-165.

Xie, F. \& Hu, G. [1997] "Spatiotemporal periodic and chaotic patterns in a two-dimensional coupled map lattice system," Phys. Rev. E55, 79-86. 